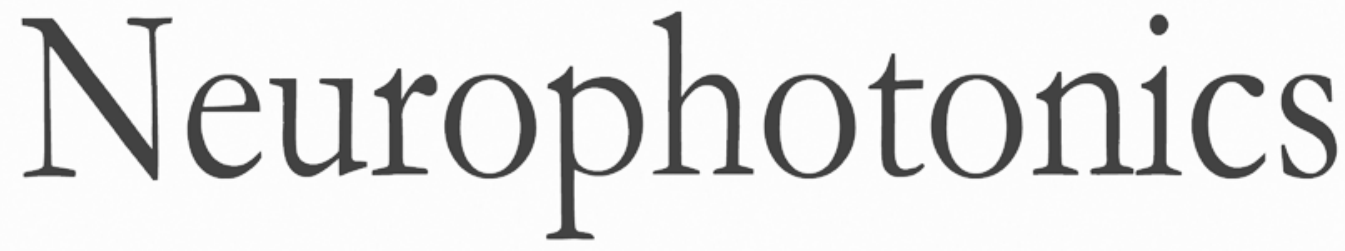

\title{
Spatiotemporal dynamics of pial collateral blood flow following permanent middle cerebral artery occlusion in a rat model of sensory- based protection: a Doppler optical coherence tomography study
}

Jiang Zhu

Aneeka M. Hancock

Li Qi

Klaus Telkmann

Babak Shahbaba Zhongping Chen Ron D. Frostig 


\title{
Spatiotemporal dynamics of pial collateral blood flow following permanent middle cerebral artery occlusion in a rat model of sensory-based protection: a Doppler optical coherence tomography study
}

\author{
Jiang Zhu, ${ }^{\mathrm{a}, \dagger}$ Aneeka M. Hancock, ${ }^{\mathrm{b}, \dagger} \mathrm{Li}$ Qi, ${ }^{a}$ Klaus Telkmann, ${ }^{c}$ Babak Shahbaba, ${ }^{\mathrm{c}}$ Zhongping Chen, ${ }^{\mathrm{a}, \mathrm{d}, \star}$ and \\ Ron D. Frostig ${ }^{b, d, e, *}$ \\ aUniversity of California Irvine, Beckman Laser Institute, Irvine, California, United States \\ bUniversity of California Irvine, Department of Neurobiology and Behavior, Irvine, California, United States \\ 'University of California Irvine, Department of Statistics, Irvine, California, United States \\ dUniversity of California Irvine, Department of Biomedical Engineering, Irvine, California, United States \\ eUniversity of California Irvine, Center for the Neurobiology of Learning and Memory, Irvine, California, United States
}

\begin{abstract}
There is a growing recognition regarding the importance of pial collateral flow in the protection from impending ischemic stroke both in preclinical and clinical studies. Collateral flow is also a major player in sensory stimulation-based protection from impending ischemic stroke. Doppler optical coherence tomography has been employed to image spatiotemporal patterns of collateral flow within the dorsal branches of the middle cerebral artery (MCA) as it provides a powerful tool for quantitative in vivo flow parameters imaging (velocity, flux, direction of flow, and radius of imaged branches). It was employed prior to and following dorsal permanent MCA occlusion ( $\mathrm{pMCAo}$ ) in rat models of treatment by protective sensory stimulation, untreated controls, or sham surgery controls. Unexpectedly, following pMCAo in the majority of subjects, some MCA branches continued to show anterograde blood flow patterns over time despite severing of the MCA. Further, in the presence of protective sensory stimulation, the anterograde velocity and flux were stronger and lasted longer than in retrograde flow branches, even within different branches of single subjects, but stimulated retrograde branches showed stronger flow parameters at $24 \mathrm{~h}$. Our study suggests that the spatiotemporal patterns of collateralbased dorsal MCA flow are dynamic and provide a detailed description on the differential effects of protective sensory stimulation. () The Authors. Published by SPIE under a Creative Commons Attribution 4.0 Unported License. Distribution or reproduction of this work in whole or in part requires full attribution of the original publication, including its DOI. [DOI: 10.1117/1.NPh.6.4.045012]

Keywords: leptomeningeal collaterals; leptomeningeal anastomoses; Doppler optical coherence tomography; cortex; neurovascular. Paper 19073RR received Jul. 15, 2019; accepted for publication Nov. 19, 2019; published online Dec. 10, 2019.
\end{abstract}

\section{Introduction}

Stroke is the fifth leading cause of death and the leading cause of long-term disability in the United States, with $88 \%$ of strokes originating from blood flow blockage known as ischemic strokes. ${ }^{1}$ The majority of cortical ischemic strokes in humans originate from blockage in the middle cerebral artery (MCA). Currently, there are only two U.S. Food and Drug Administration-approved treatments: (1) an injectable drug [recombinant tissue plasminogen activator (rtPA)] and (2) a procedure known as stent retrieval thrombectomy. Despite the success of these treatments, there still remains a sizable proportion of patients for whom substantial reperfusion cannot be achieved..$^{2-4}$ Therefore, in recent years, there has been an active search for alternative or complementary protection strategies to these treatments or as stand-alone treatments.

Using a rat model of ischemic stroke achieved by dorsal permanent middle cerebral artery occlusion (pMCAo), we have repeatedly demonstrated that delivery of a whisker stimulation

*Address all correspondence to Ron D. Frostig, E-mail: rfrostig@uci.edu; Zhongping Chen, E-mail: z2chen@hs.uci.edu

tThese authors contributed equally to this work. during a 2-h window following pMCAo directly evokes neuronal activity within the MCA ischemic territory. Such activation results in functional and structural protection from impending ischemic stroke, findings that were independently replicated in other labs. ${ }^{5-10}$ Further, our findings $s^{11-18}$ (reviewed in Refs. 19-21) have demonstrated that such stimulus-based protection is achievable in anesthetized rats, unrestrained behaving rats, and in old rats. Such protection depends on sensory activation of the ischemic MCA territory even in whiskerless rats, and it seems to hold for at least 4 months. This protection critically depends on functional pial collaterals (leptomeningeal collaterals or leptomeningeal anastomoses) that connect the MCA with the other two major cortical arteries (anterior cerebral artery and posterior cerebral artery) that act as alternative arterial blood supply sources of retrograde blood flow into the occluded MCA. Collateral-based retrograde flow starts following pMCAo and can sustain the cortex for only about $2 \mathrm{~h}$ without sensory stimulation. Delivering whisker stimulation immediately after pMCAo, or at 1 or $2 \mathrm{~h}$ following pMCAo protects the ischemic MCA territory by enhancing the retrograde-based flow into the occluded MCA resulting in protective reperfusion of the ischemic MCA territory. Collectively, our collateral-based findings further support and extend the emerging field known as "collateral therapeutics"-experimental stroke protection therapies 
that are applied in emergency departments during the hyperacute state of ischemic stroke by enhancing protective blood flow through collateral recruitment (for recent preclinical and clinical reviews, see Refs. 22-26). Common stroke risk factors, including metabolic syndrome and age, are also associated with poor pial collateral flow in humans and conversely, good collateral flow is associated with improved likelihood of major reperfusion, reduced infarct size, and more favorable outcomes. Further, as was recently reported, stroke patients with good collaterals could still have salvaged penumbra by recanalization even at 6 to $16 \mathrm{~h}$ after the first signs of stroke, ${ }^{27}$ a surprising finding because previous recommendations emphasized that only fast intervention could salvage cortical tissue in all patients. Indeed, a recent study concluded that penumbral salvage and infarct growth in humans are less time-dependent and more a measure of collateral flow. ${ }^{28}$ However, despite the growing recognition of the importance of collateral therapeutics, this research field is still in its nascent phase because collateral function and its resulting flow patterns are poorly understood.

To further the study of collateral flow patterns within dorsal MCA branches, we have employed Doppler optical coherence tomography (DOCT). DOCT is a high-resolution, quantitative in vivo imaging technique that can measure absolute velocity and flux of blood flow within cerebral blood vessels that are up to 2 to $3 \mathrm{~mm}$ deep; for recent reviews, see Refs. 29 and 30. DOCT can target a specific location within the blood vessel from which to quantify velocity and flux. ${ }^{31}$ In addition, DOCT informs about the direction of blood flow, a crucial parameter in the study of retrograde flow patterns from pial collaterals.

The present study employed DOCT to study collateral-based flow patterns within the dorsal branches of the occluded MCA. We were able to determine velocity, flux, direction of blood flow, and radius of dorsal MCA branches prior to and continuously for $3 \mathrm{~h}$ after pMCAo, and again at $24 \mathrm{~h}$ after pMCAo within the same rat in three experimental groups: treated (with $2 \mathrm{~h}$ of protective sensory stimulation following pMCAo), untreated (without protective sensory stimulation), and sham surgery controls.

\section{Methods}

All procedures were in compliance with U.S. National Institutes of Health (NIH) guidelines and approved by UC Irvine Animal Care and Use Committee (Protocol No. 1997-1608, assurance ID No. A3416.01).

\subsection{Subjects and Surgical Preparation}

Nineteen experimental subjects, 295 to $400 \mathrm{~g}$ (3 to 4 months of age) male Sprague Dawley rats (Charles River Laboratories, Wilmington, Massachusetts), were individually housed in standard cages. At the beginning of each experiment, subjects were injected intraperitoneally with a Nembutal bolus $(55 \mathrm{mg} / \mathrm{kg}$ b.w.). Supplemental injections of Nembutal (27.5 mg/kg b.w.) were given as necessary. After resection of soft tissue, an $\sim 6.5 \times$ $8 \mathrm{~mm}^{2}$ "imaging" area of the skull over the left primary somatosensory cortex (rostromedial corner positioned $\sim 1 \mathrm{~mm}$ caudal and $2 \mathrm{~mm}$ lateral from bregma) was thinned to $\sim 150 \mu \mathrm{m}$ using a dental drill. Five percent dextrose $(3 \mathrm{~mL})$ and atropine $(0.05 \mathrm{mg} / \mathrm{kg}$, b.w.) were administered at the beginning of the experiment and every $6 \mathrm{~h}$ until the animal was returned to its home cage (the first day of each experiment typically lasted 7 to $9 \mathrm{~h}$ and the second day, at $24 \mathrm{~h}$ postocclusion, typically lasted 2 to $4 \mathrm{~h}$ ). Body temperature was measured via a rectal probe and maintained at $37^{\circ} \mathrm{C}$ by a self-regulating thermal blanket. After completion of the experiment, all animals were returned to their home cages and allowed to recover. All subjects received flumeglumine $(2.5 \mathrm{mg} / \mathrm{kg} \mathrm{b}$.w. $)$ at the end of surgery, and the health of the animals was monitored hourly.

Baseline data collection was followed by pMCAo of the M1 segment: double ligature and transection of the stem (primary branch of the middle cerebral artery segment, just distal to the lenticulostriate branch) of the left proximal middle cerebral artery. $^{15}$

\subsection{Overview}

DOCT experimental timelines are summarized in Fig. 1. Using a subject design that is identical to our previous studies, 19 subjects were randomly assigned to a $+0 \mathrm{~h}$ group, i.e., these animals were stimulated immediately $(0 \mathrm{~h})$ after pMCAo, an identical no stimulation control group, and a sham surgical control group. Baseline DOCT was collected for all subjects prior to pMCAo. All $+0 \mathrm{~h}(n=6)$ and no stimulation control $(n=8)$ subjects then received pMCAo, which was followed by immediate postocclusion whisker stimulation lasting $2 \mathrm{~h}$ followed by a quiet period lasting $1 \mathrm{~h}$ for $+0 \mathrm{~h}$ subjects, or a quiet period lasting $3 \mathrm{~h}$ for no stimulation controls (light and sound were minimized during this period). Postocclusion whisker stimulation consisted of $1 \mathrm{~s}$ of $5-\mathrm{Hz}$ deflections of a single whisker (whisker C2). This stimulation was intermittently delivered (with random interstimulus intervals averaging $21 \mathrm{~s}$ ) 256 times, totaling $4.27 \mathrm{~min}$ of stimulation, over the course of $2 \mathrm{~h}^{11}$ Surgical shams $(n=5)$ underwent identical surgery to that of $+0 \mathrm{~h}$ subjects with the suture needle and thread passing under the MCA, but sutures were not tied around the MCA, leaving the blood vessel intact. Sham surgery was immediately followed by whisker stimulation lasting $2 \mathrm{~h}$ followed by a quiet period lasting $1 \mathrm{~h}$. After the measurements of $3 \mathrm{~h}$, all rats were placed back in their home cages for recovery until $24 \mathrm{~h}$ postocclusion at which point a follow-up assessment was conducted. Rats were then euthanized for histological assessment (see below for detailed methodology and experimental design). All DOCT measurements were blindly performed in regard to the experimental groups.

\subsubsection{Histology}

At the conclusion of each experiment, rats were euthanized with sodium pentobarbital ( 2 to $3 \mathrm{~mL}$, intraperitoneally), the brain was removed, sectioned into $2-\mathrm{mm}$ coronal slices, and incubated in $2 \%$ 2,3,5-triphenyltetrazolium chloride (TTC) at $37^{\circ} \mathrm{C}$ for $20 \mathrm{~min}$ in the dark. ${ }^{32}$ The edema-corrected infarct volume was quantified by an observer blind to the experimental condition.

\subsection{Doppler Optical Coherence Tomography}

A swept source-based OCT system with a central wavelength of $1310 \mathrm{~nm}$ and an A-line speed of $50 \mathrm{kHz}$ (SSOCT-1310, Axsun Technologies Inc., Billerica, Massachusetts) was used for imaging of the vascular networks and calculation of flow velocities, which is shown in Fig. 2. The light power from the swept source was split $90 \%$ into the sample arm and $10 \%$ into the reference arm by a $2 \times 2$ fiber optic coupler with a coupling ratio of $90 / 10$. In the sample arm, the light reached the cerebral cortex through a broadband fiber optic circulator, a collimator, a two-axial galvoscanning mirror for two-dimensional (2-D) scanning, and a scan lens with a $45-\mathrm{mm}$ focusing length. In the reference arm, a 
Sham surgical control subjects

$3 \mathrm{~h}$

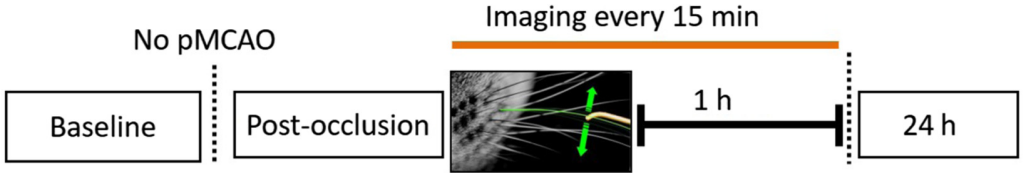

Non-stimulated control subjects

$3 \mathrm{~h}$

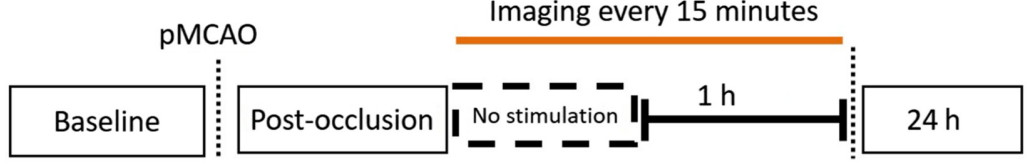

$3 \mathrm{~h}$

+0 $\mathrm{h}$ subjects

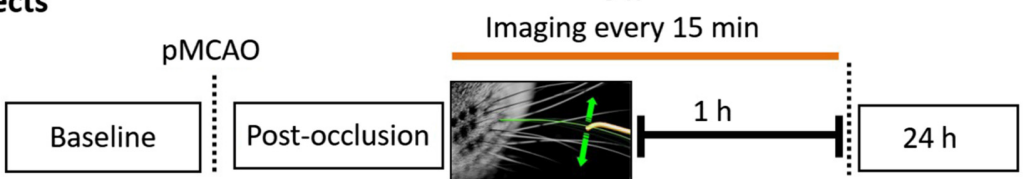

(a)

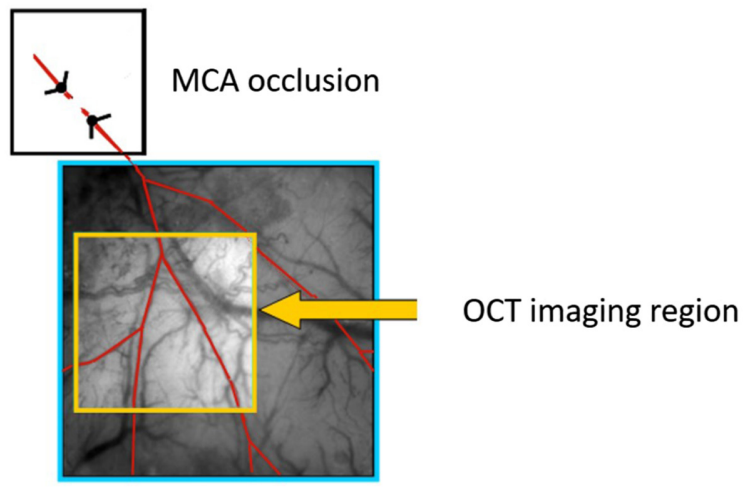

(b)

Fig. 1 (a) Experimental timelines of OCT imaging. Three experimental subjects were imaged, including sham surgical control subjects, pMCAo group without whisker stimulation (no-stimulation control subjects), and pMCAo group with whisker stimulation ( $+0 \mathrm{~h}$ subjects). (b) Doppler OCT imaging window that was used in the experiments; MCA imaged branches are highlighted in red. Note that the MCA occlusion site (the M1 section of the MCA) is always outside the DOCT imaging window.

mirror reflected the light after the light passed through a broadband fiber optic circulator, a collimator, and a focusing lens. The light from the reference arm and from the sample arm interfered in a $2 \times 2$ fiber optic coupler with a coupling ratio of 50/50, and then was split 50\% and 50\% into two inputs of a dual-balanced

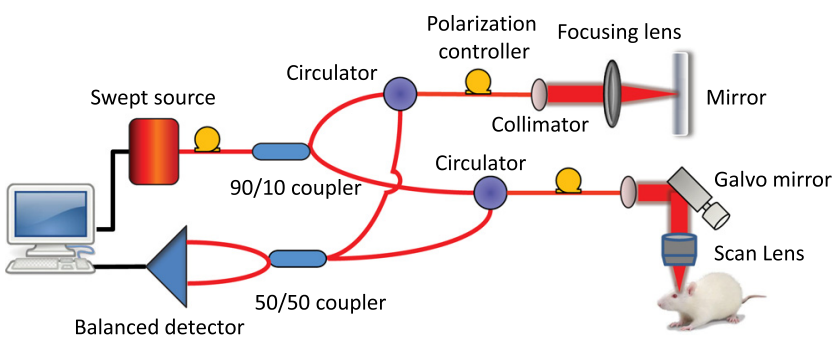

Fig. 2 Schematic of OCT system. The OCT system is based on a swept source with a wavelength of $1310 \mathrm{~nm}$ and A-line speed of $50 \mathrm{kHz}$. detector. Signals from the detector were collected by a 12-bit PCI express digitizer, and the galvo mirror was controlled by a data acquisition board with two 16-bit analog outputs for 2-D scanning. A visual C++ platform-based software running on 64-bit Windows 7 operation systems was used to control the galvo mirror, acquire the OCT data, and analyze the data. This system had a lateral resolution of $22.1 \mu \mathrm{m}$ and an axial resolution of $7.6 \mu \mathrm{m}$ in air, and the minimum A-line sampling interval was $0.02 \mathrm{~ms}$. For the calculation of flow velocities, 100 repeats were performed at the selected B-scan position with 2048 Alines. During the microvascular mapping, the scanning area contained 1024 or 2048 A-lines in each of 256 B-scans. Each A-line contained 512 sampling spots.

We used a phase-resolved Doppler OCT method for quantification of flow velocities and used an OCT intensity-based Doppler variance (IBDV) method for mapping of microvascular networks. The Doppler phase method can provide accurate flow velocities and flow direction combining the Doppler principle 
and OCT signals; however, it suffers from phase wrapping and Doppler angles should be determined before calculation of flow velocities. The IBDV method is not affected by phase wrapping and has the ability to image microvascular networks with simpler data processing, and thus is suitable for the qualitative comparison of the microvasculature.

The Doppler phase method was used to calculate the flow velocity $V$ as $29,30,33$

$V \cdot \cos (\theta)=\frac{\Delta \phi \cdot \lambda_{0}}{4 \pi \cdot n \cdot \Delta T}$

where $\theta$ is the Doppler angle between the OCT beam and a detected vessel, which can be determined by three-dimensional rendering of the vascular networks, $\lambda_{0}$ is the central wavelength of the light source, $n$ is the refractive index, $\Delta T$ is the sampling interval, and $\Delta \phi$ is the phase shift between different A-lines. The parameters of $\lambda_{0}$ and $n$ are $1310 \mathrm{~nm}$ and 1.4, respectively. From this equation, $V \cdot \cos (\theta)$ is a component of the flow velocity along the OCT beam direction and $\Delta \phi / \Delta T$ is the rate of phase shift change. If $V$ is too low, $\Delta \phi$ will be too small to measure. In order to obtain a detectable value of $\Delta \phi, \Delta T$ should be increased for the measurement of a low flow velocity. If $V$ is too high, $\Delta \phi$ will wrap and cannot be measured accurately. By changing the sampling interval $\Delta T$, the measurement range of flow velocities can be adjusted for the Doppler OCT method. In order to calculate the flow velocity in a wider range, A-line sampling intervals of $0.02,0.04,0.10,0.20$, and $0.40 \mathrm{~ms}$ were applied. For the measurement of the phase shift $\Delta \phi$ from complex OCT signals, the following equation can be utilized

$$
\Delta \phi_{j, i}=\tan ^{-1}\left[\frac{\operatorname{Im}\left(A_{j, i} \cdot A_{j+1, i}^{*}\right)}{\operatorname{Re}\left(A_{j, i} \cdot A_{j+1, i}^{*}\right)}\right],
$$

where $A_{j, i}$ is the complex signal at the $j$ 'th A-line and $i$ 'th depth obtained from the OCT data, and $\operatorname{Im}()$ and $\operatorname{Re}()$ are the imaginary component and real component of the complex signal, respectively. The flux can be calculated by incorporating the measurements of the diameters of the vessels and the quantification of the flow velocities from Doppler OCT images, as Flux $=V \cdot \pi \cdot(D / 2)^{2}$, where $V$ is the flow velocity and $D$ is the lumen diameter.

For the mapping of microvascular networks, an IBDV is calculated, which utilizes amplitude correlation between A-lines to visualize the vascular networks instead of the phase difference, which is described by the following equation $29,30,33-35$

$$
\sigma_{j, i}^{2}=1-\frac{2 \cdot\left|A_{j, i} \cdot A_{j+1, i}^{*}\right|}{\left|A_{j, i}\right|^{2}+\left|A_{j+1, i}\right|^{2}},
$$

where $\sigma_{j, i}^{2}$ is the standard deviation and is only affected by amplitude differences among A-lines. With an increase of the sampling interval between adjacent A-lines, the value of $\sigma_{j, i}^{2}$ will increase until saturation. For the detection of low velocity blood flow, the sampling interval is progressively increased.

\subsubsection{Statistics}

Regression analysis: in the following, let $v_{t, i}, f_{t, i}$, and $r_{t, i}$ denote measurements of blood flow velocities, flux, and the radius at time point $t$ and branch $i$, respectively. Specifically, these measurements are continuously obtained at baseline, occlusion, and for $3 \mathrm{~h}$ in 15-min intervals following occlusion. A final measurement corresponds to $24 \mathrm{~h}$ after occlusion. Thus, we treat the measurements for each branch as a time series.

We propose the following analysis to determine potential significant effects of the stimulation. Let $v_{\mathrm{occ}, i}, f_{\mathrm{occ}, i}$ and $r_{\mathrm{occ}, i}$ denote the velocity, flux, and radius, respectively, at occlusion and branch $i$. Since stimulation is received after occlusion, we consider the changes relative to occlusion, namely,

$\widetilde{v_{t, l}}=v_{t, i}-v_{\mathrm{occ}, i}$

Note that $\widetilde{v_{\mathrm{occ}, l}}=0$ by construction. Consider the model

$\widetilde{v_{t, l}}=\beta_{0}+\beta_{1} 1_{\mathrm{G}}(i)+\varepsilon_{t, i}$,

for $t \in\{15,30, \cdots, 180\} \min$. The dummy variable $1_{G}(i)$ takes the value 1 if subject $i$ belongs to the stim group and 0 otherwise and distinguishes between different treatments. Finally, $\varepsilon_{t, i}$ is a zero mean error process with

$\operatorname{cov}\left(\varepsilon_{t_{1}, i}, \varepsilon_{t_{2}, j}\right)=0 \quad$ for $i \neq j$

Specifically, measurements obtained from different branches are assumed to be independent whereas errors within branches may be correlated. No further specific assumptions about the errors have to be made (see, e.g., Refs. 36 and 37). The parameter $\beta_{1}$ determines the difference in means between the two treatments. Estimates and $p$-values are obtained by linear regression adjusted for cluster robust standard errors where each branch serves as a cluster. The same model is applied to the flux and radius measurements. Using the statistical software $\mathrm{R}$ (based on $\mathrm{R}$ version 3.5.1), we could estimate the coefficients $\beta_{0}$ and $\beta_{1}$ using the $g l m$ function. Applying the result to coef_test in the clubSandwich package ${ }^{38}$ and clustering by branches led to adjusted standard errors and more reliable and conservative $p$-values. Earlier attempts to include "time" and "radius" in the time-series regression equation showed that the addition of these variables did not provide any new information, and therefore, these variables were dropped except in one case where the time variable added new significant information (see below for the case of "radius anterograde").

We use Welch $t$-tests (function t.test in R) whenever the normality assumption was confirmed by a Shapiro-Wilk normality test (function Shapiro.test in R). If normality had to be rejected, paired differences were tested by a Wilcoxon signed-rank test and unpaired differences by a Mann-Whitney U test (the latter two tests use the same function in R, namely the Wilcox. test, for the signed-rank test paired=TRUE is applied and for the MannWhitney paired=FALSE is applied). All results are shown as mean and standard error and retrograde values have been assigned a negative value.

\section{Results}

\subsection{Validation of Flow Measurements using OCT}

In order to validate flow measurements using DOCT, a flow phantom was prepared in which $5 \%$ intralipid solution was infused into a transparent polymer tube with an inner diameter of $0.8 \mathrm{~mm}$ by a syringe pump, which is shown in Fig. 3(a). The polymer tube was fixed on a stage to keep a Doppler angle of 70 deg between the flow and OCT beam. The flow velocities were controlled by the syringe pump from 0 to $70 \mathrm{~mm} / \mathrm{s}$. 


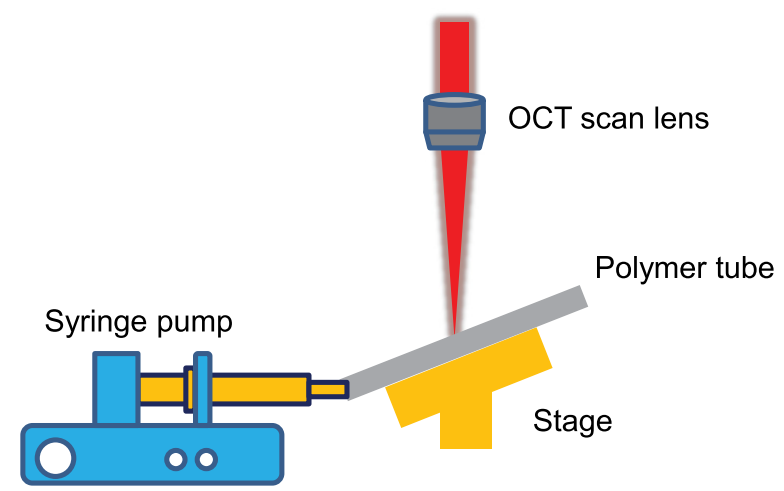

(a)

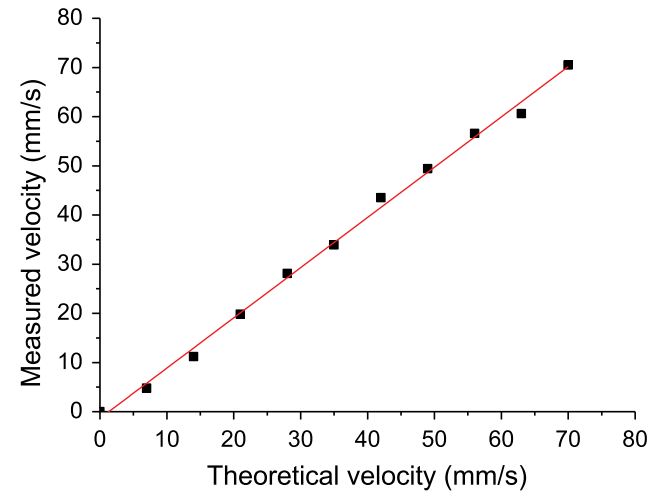

(b)

Fig. 3 Flow measurements using a flow phantom. (a) System setup for the flow measurements. (b) Comparison between theoretical flow velocities controlled by a syringe pump and measured phase shifts by Doppler OCT imaging.

The measured phase shifts from the Doppler OCT imaging were compared with the injection velocities, and there is a linear relationship with $R^{2}>0.99$ between the measured phase shifts and injection velocities, see Fig. 3(b).

In order to assess the ability of the DOCT system for in vivo blood blow measurements, we measured the spatial distributions and temporal changes of blood flow in major MCA branches of a rat cortex. Figure 4 shows blood flow measurements in a rat at the baseline. IBDV projection shows a microvascular network in the MCA region of a rat brain in Fig. 4(a). Three major MCA branches were selected for the blood flow measurements, which are shown in Fig. 4(a). The cross-sectional OCT image and phase shift image are shown in Figs. 4(b) and 4(c). Three major MCA branches can be identified clearly for the flow quantification under the dura. The spatial distribution of flow velocities is analyzed in Fig. 4(d). The flow velocities are not uniform in the vessels where the flow velocity is larger in the central region than in the peripheral region. For the analysis of flow velocities over time in central regions of vessels, Doppler OCT images at one location were acquired employing 100 repeats with a frame interval of $0.02 \mathrm{~s}$, as shown in Fig. 4(e). The changes of flow velocities due to the heartbeat pulses are also recorded as part of the measurements, indicating that Doppler OCT measurements have high temporal resolution for the quantification of blood flow in rat brains. From Fig. 4(e), the pulse frequency is $\sim 323$ cycles/ $\min$ for this specific rat. In the following measurements, we calculated the flow velocity in the central region of each vessel with an average of 100 repeats to eliminate the heartbeat influence.

\subsection{Flow Measurements in Sham Surgical Control Subjects}

Measurements were acquired from a total of 12 MCA branches obtained from five rats where two to three major MCA dorsal branches were measured within each subject. At baseline, flow velocities ranged from 11.1 to $31.8 \mathrm{~mm} / \mathrm{s}$ with a mean of $20.3 \pm 0.46 \mathrm{~mm} / \mathrm{s} ; \quad$ flux values ranged from 0.1 to $0.6 \mathrm{~mm}^{3} / \mathrm{s}$ with a mean of $0.39 \pm 0.015 \mathrm{~mm}^{3} / \mathrm{s}$ and the radii ranged from 47 to $95.5 \mu \mathrm{m}$ with a mean of $75.5 \pm 1.4 \mu \mathrm{m}$.

After confirming normality, a Welch $t$-test demonstrated that there was an increase between baseline and the mean of the time period from occlusion to $180 \mathrm{~min}$ in velocity and flux $(d f=11$, $p=0.01$, and $p=0.04$, respectively), while there was no difference for the radius ( $d f=11, p=0.34)$ (Fig. 5).

\subsection{Flow Measurements in pMCAo Subjects}

\subsubsection{General analysis design}

Following pMCAo, we expected to reveal only collateral-based retrograde flow in all major MCA branches as the MCA was severed at the M1 level, and therefore, there was no clear source that could supply anterograde flow. Unexpectedly, flow direction remained the same (i.e., anterograde direction) in some major MCA branches, but reversed (i.e., retrograde direction) in others. Anterograde and retrograde flows were imaged in different MCA branches within the same subject in the majority of cases [five out of six (83\%) in the +0 h group; seven out of eight $(87.5 \%)$ in the no-stimulus group]. Therefore, to further analyze these data, two additional groups were added: anterograde flow direction versus retrograde flow directions. Consequently, our analysis was applied to four groups: $+0 \mathrm{~h}$ anterograde (10 branches), $+0 \mathrm{~h}$ retrograde (6 branches), no-stimulation anterograde (6 branches), and no-stimulation retrograde (10 branches).

\subsubsection{Baseline analysis}

At baseline, flow velocities ranged from 5.36 to $38.87 \mathrm{~mm} / \mathrm{s}$ with a mean of $14.05 \pm 0.2 \mathrm{~mm} / \mathrm{s}$; flux values ranged from 0.02 to $1.19 \mathrm{~mm}^{3} / \mathrm{s}$ with a mean of $0.214 \pm 0.0069 \mathrm{~mm}^{3} / \mathrm{s}$ and the radii ranged from 35 to $98.6 \mu \mathrm{m}$ with a mean of $62.5 \pm 0.54 \mu \mathrm{m}$. When further analyzed in all subgroups, baseline flow velocities ranged from 6.5 to $38.9 \mathrm{~mm} / \mathrm{s}$ with a mean of $16.1 \pm 8.2 \mathrm{~mm} / \mathrm{s}$ for $+0 \mathrm{~h}$ subjects and ranged from 5.4 to $19.5 \mathrm{~mm} / \mathrm{s}$ with a mean of $11.9 \pm 3.8 \mathrm{~mm} / \mathrm{s}$ for no-stimulation control subjects. Flux ranged from 0.03 to $1.19 \mathrm{~mm}^{3} / \mathrm{s}$ with an average of $0.28 \pm 0.017 \mathrm{~mm}^{3} / \mathrm{s}$ for $+0 \mathrm{~h}$ subjects and ranged from 0.02 to $0.37 \mathrm{~mm}^{3} / \mathrm{s}$ with a mean of $0.15 \pm 0.0066 \mathrm{~mm}^{3} / \mathrm{s}$ for no-stimulations subjects; radii ranged from 38.4 to $98.6 \mu \mathrm{m}$ with a mean of $66.8 \pm 1.13 \mu \mathrm{m}$ for $+0 \mathrm{~h}$ subjects, and from 35 to $84 \mu \mathrm{m}$ with a mean of $57.9 \pm 0.97 \mu \mathrm{m}$ for no-stimulation subjects. 
IBDV projection

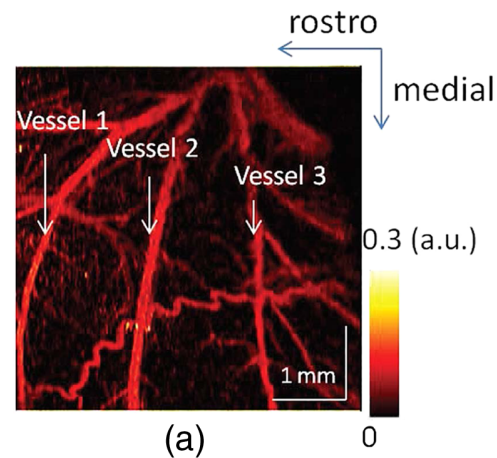

(a)

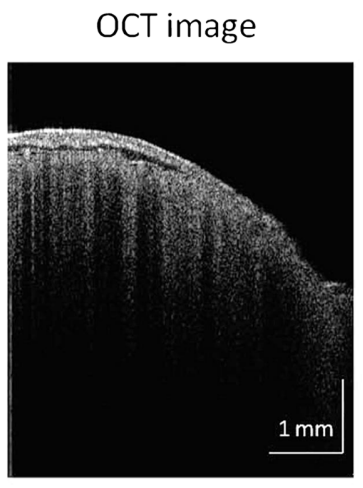

(b)

Phase-resolved Doppler image

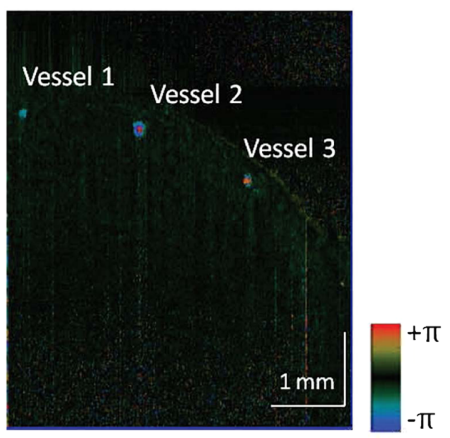

(c)

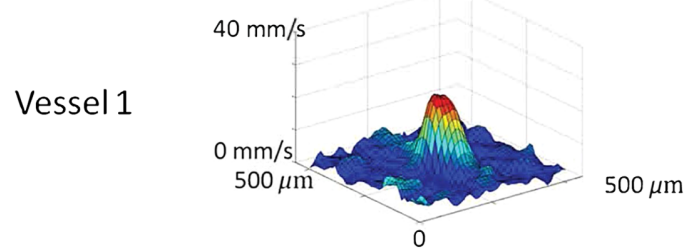

Vessel 2

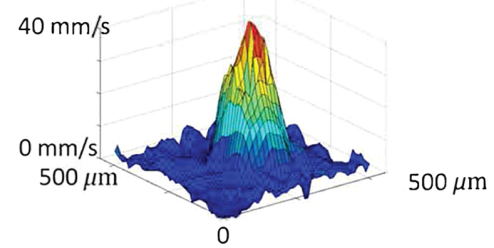

Vessel3

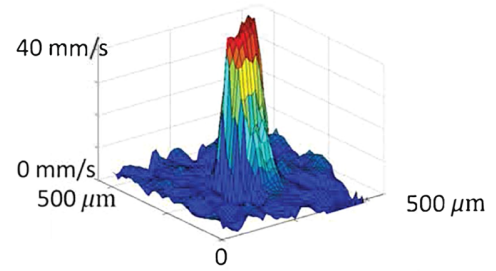

(d)

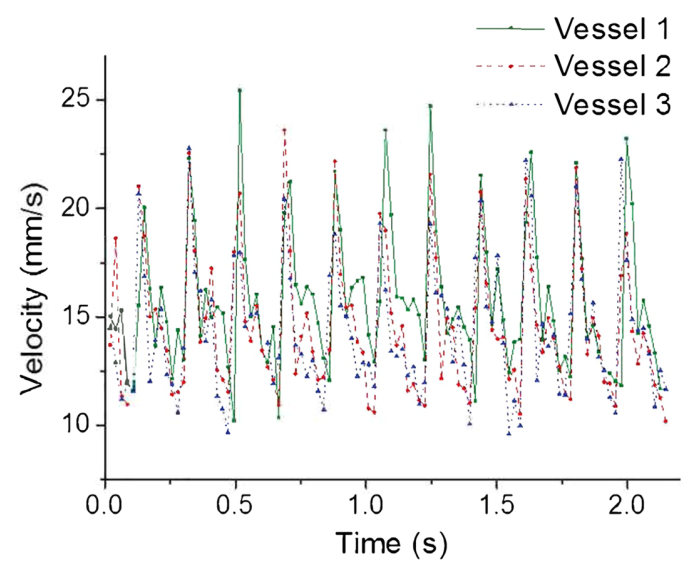

(e)

Fig. 4 Blood flow measurements in a sham surgical control subject. (a) IBDV projection from OCT imaging shows a microvascular network in the MCA region of a rat brain. A sampling A-line interval of $0.40 \mathrm{~ms}$ was used. (b) Cross-sectional OCT image. (c) Phase-resolved Doppler OCT cross-sectional scan for flow quantification. Three major MCA branches can be identified clearly under the dura mater. A sampling interval of $0.02 \mathrm{~ms}$ was used. (d) Distribution of flow velocities in three branches at one time. (e) Flow velocities in three branches over time. The changes of flow velocities were caused by the heartbeats.

\subsubsection{Following PMCAO}

Following pMCAo, flow velocity dropped from a mean of $14.05 \mathrm{~mm} / \mathrm{s}$ at baseline to $1.6 \mathrm{~mm} / \mathrm{s}$ at the occlusion time point (11.4\% of baseline) and flux dropped from 0.214 to $0.017 \mathrm{~mm}^{3} / \mathrm{s}$ ( $8.2 \%$ of baseline). Radius mean increased from 62.5 to $62.8 \mu \mathrm{m}$ (100.4\% of baseline). When further analyzed in all subgroups, following pMCAo, flow velocity mean dropped in the $+0 \mathrm{~h}$ group from $16.1 \mathrm{~mm} / \mathrm{s}$ at baseline to $1.99 \mathrm{~mm} / \mathrm{s}$ at occlusion (12.4\% of baseline) and flux mean dropped from 0.28 to $0.02 \mathrm{~mm}^{3} / \mathrm{s}$ ( $8.23 \%$ of baseline). Radius mean increased from 66.8 to $67.9 \mu \mathrm{m}$ (101.5\% of baseline). For the no-stimulus group, flow velocity dropped from a mean of $11.9 \mathrm{~mm} / \mathrm{s}$ at baseline to $1.19 \mathrm{~mm} / \mathrm{s}$ (10\% of baseline) and flux dropped from a mean of $0.15 \mathrm{~mm}^{3} / \mathrm{s}$ at baseline to 0.012 (8.0\% of baseline), whereas radius decreased from a mean of 57.9 to $57.4 \mu \mathrm{m}(99 \%$ of baseline) [Figs. 6(a)-6(h)]. Since the Shapiro-Wilk test showed that flux and velocity for $+0 \mathrm{~h}$ subjects cannot be assumed to be normally distributed, a Mann-Whitney U test was employed to show that there was no difference between $+0 \mathrm{~h}$ and no-stimulation velocities $(p=0.11)$ and flux $(p=0.07)$ at baseline. For the radii, normality was affirmed, and a Welch 


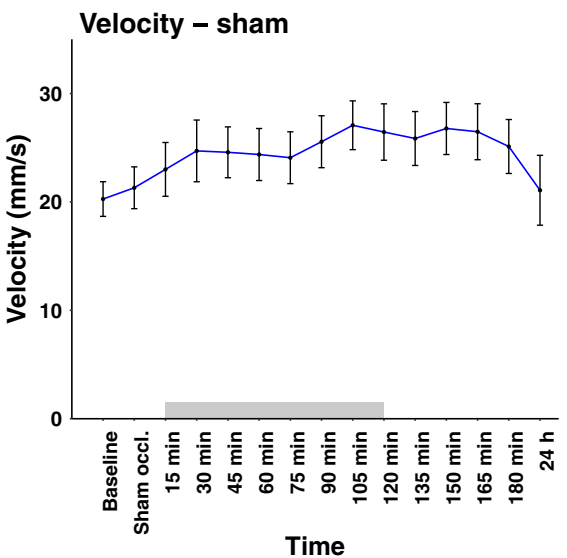

(a)

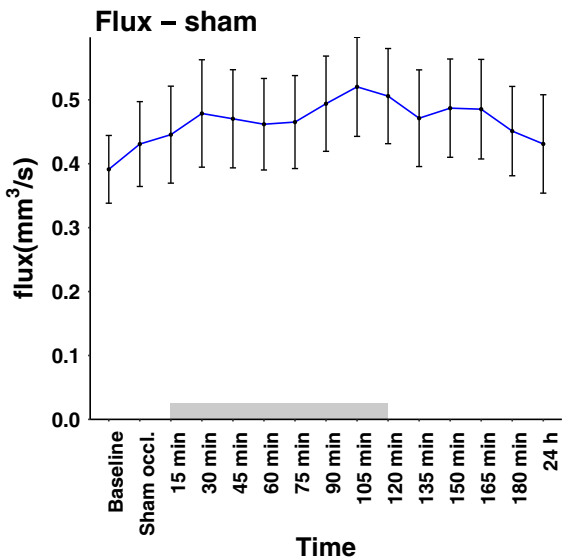

(b)

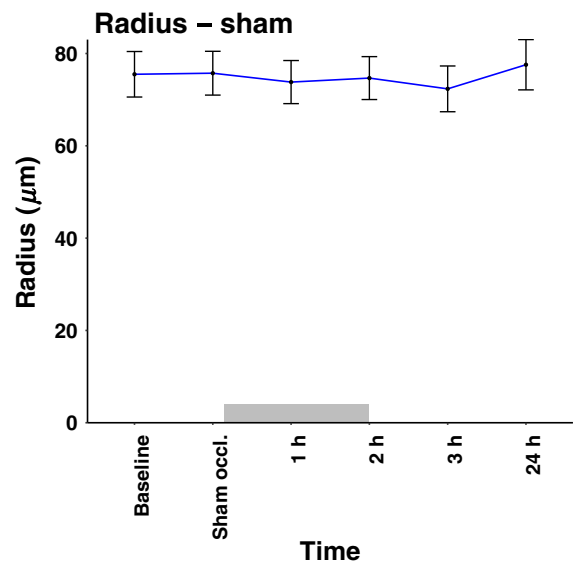

(c)

Fig. 5 Sham-surgery flow parameters and radii in the presence of sensory stimulation. In the presence of stimulation (denoted by a gray rectangle above the $x$-axis) as compared to presham-pMCAo there was (a) an increase in velocity as analyzed for the 15 to 180 min time points period, (b) increase in flux, but (c) no change in radii of the imaged branches.

$t$-test showed that there was no difference between the groups either $(d f=30, p=0.15)$.

Since flow patterns at baseline in both pMCAo groups were only anterograde, and therefore, not clearly relevant to the combination of anterograde and retrograde flow patterns postpMCAo, we searched for a new post-pMCAo common baseline point. The occlusion time point seemed optimal. Indeed, at the occlusion time point, a Welch $t$-test (after checking normality by a Shapiro-Wilk test) demonstrated that there was no difference in the absolute mean values of $+0 \mathrm{~h}(1.99 \pm 0.11 \mathrm{~mm} / \mathrm{s})$ versus nonstimulated $(1.19 \pm 0.04 \mathrm{~mm} / \mathrm{s})$ branches $(d f=24$, $p=0.12$ ) with a difference of $0.80 \pm 0.50 \mathrm{~mm} / \mathrm{s}$. Even when further divided to retrograde versus anterograde flow directions at the occlusion time point, there was no difference between $+0 \mathrm{~h}$ versus no-stimulation branches at the occlusion point as a two-sided Welch $t$-test showed no difference between $+0 \mathrm{~h}$ retrograde flow branches mean $(-2.19 \pm 0.21 \mathrm{~mm} / \mathrm{s})$ versus mean nonstimulated $(-1.2 \pm 0.06 \mathrm{~mm} / \mathrm{s})$ retrograde branches $(d f=10, p=0.19)$ with a difference of $1.0 \pm$ $0.71 \mathrm{~mm} / \mathrm{s}$. For anterograde flow direction, no difference was found between mean $+0 \mathrm{~h}$ branches $(1.49 \pm 0.2 \mathrm{~mm} / \mathrm{s})$ versus mean no-stimulation $(1.18 \pm 0.09 \mathrm{~mm} / \mathrm{s})$ branches $(d f=8$, $p=0.61$ ) with a difference of $0.30 \pm 0.55 \mathrm{~mm} / \mathrm{s}$. It was, therefore, determined that the occlusion point could serve as a new optimal reference point for further analysis as shown in Fig. 7.

Since the statistical analysis employed in this study assumes sampling at equal time intervals, detailed analysis of these experimental groups was performed only from the occlusion point up to and including the $180 \mathrm{~min}$ postocclusion point, leaving the 24$\mathrm{h}$ point for a separate analysis (see below). Results of the regression analysis in combination with the coefficient test of flow parameters are quantified as differences from occlusion time point continuously up to and including the 180-min time point. The results are separately described for velocity, flux, and radius.

Velocity. To test the congruence of the current results with our previous laser speckle imaging (LSI) findings that were agnostic to flow direction, absolute values of anterograde and absolute values of retrograde branches were pooled together and the analysis revealed a clear mean difference between $+0 \mathrm{~h}$ and nonstimulated branches of $0.56 \pm 0.17 \mathrm{~mm} / \mathrm{s}(d f=30$, $p=0.002$ ), Fig. 7(a). Anterograde blood flow: mean for $+0 \mathrm{~h}$ stimulated branches was faster by $1.04 \pm 0.385 \mathrm{~mm} / \mathrm{s}$ $(d f=11, p=0.02)$ versus the mean of the nonstimulated branches as shown in Fig. 7(b) (note the larger standard error of the mean for the stimulated branches). Retrograde blood flow: the analysis revealed that the presence of the stimulus had no clear effect on +0 h stimulated branches' mean compared to the no-stimulation branches' mean $-0.22 \pm 0.21 \mathrm{~mm} / \mathrm{s}(d f=10$, $p=0.3$ - probably due to an increase in variability in the stimulated branches compared to no-stimulation branches [Fig. 7(c)]. However, when only the first $45 \mathrm{~min}$ of the velocity were analyzed, there was a strong tendency for the $+0 \mathrm{~h}$ stimulated branches to be faster, but it did not reach significance $-0.475 \pm 0.227 \mathrm{~mm} / \mathrm{s}(d f=10, p=0.06)$. Such potential early difference disappeared within the 60 to $180 \mathrm{~min}$ time interval following occlusion $-0.143 \pm 0.229 \mathrm{~mm} / \mathrm{s} \quad(d f=10$, $p=0.54)$ [Fig. 7(c)]. Finally, no differences were found between the absolute values of anterograde versus retrograde velocity for the $+0 \mathrm{~h}(-0.634 \pm 0.386 \mathrm{~mm} / \mathrm{s} ; \quad d f=10$, $p=0.12$ ) or for the absolute values of anterograde versus retrograde in the no-stimulation velocity $(-0.025 \pm 0.08 \mathrm{~mm} / \mathrm{s}$; $d f=11, p=0.76)$. Collectively, these findings show a clear general enhancing effect of sensory stimulation on velocity, an effect that can be mostly attributed to anterograde vessels despite an increase in their variability. When stimulation was present, anterograde velocity was faster in the stimulated branches versus the nonstimulated branches, and this effect lasted after termination of the stimulus. The retrograde flow branches tended to be faster within the first $45 \mathrm{~min}$. See Fig. 8 for examples of changes in the speed and direction of blood flow velocities in a representative no-stimulation subject and in a stimulation subject.

Similar results were obtained for flux measurements. Following the pooling of the absolute values of the anterograde and retrograde values, the difference between stimulated and nonstimulated flux means was clear, $0.008 \pm 0.002 \mathrm{~mm}^{3} / \mathrm{s}$ $(d f=30, p=0.007)$; Fig. 7(d). For anterograde flux, there was a difference between the means of the $+0 \mathrm{~h}$ and nonstimulated branches of $0.013 \pm 0.005 \mathrm{~mm}^{3} / \mathrm{s}(d f=11, p=0.03)$; Fig. 7(e). For retrograde blood flow, there was no mean difference between flux means in the $+0 \mathrm{~h}$ versus no-stimulation 


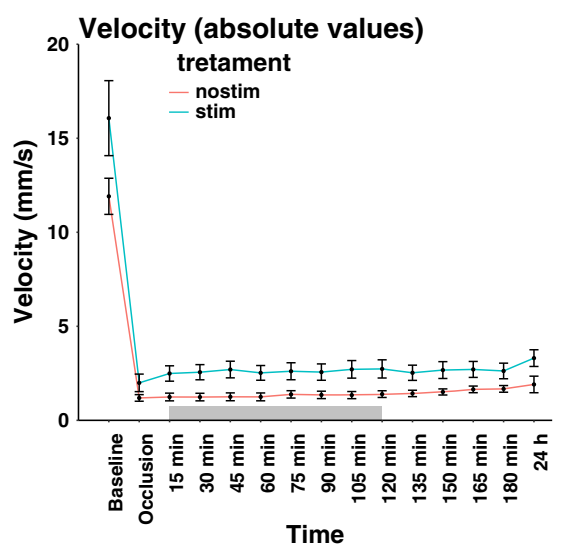

(a)

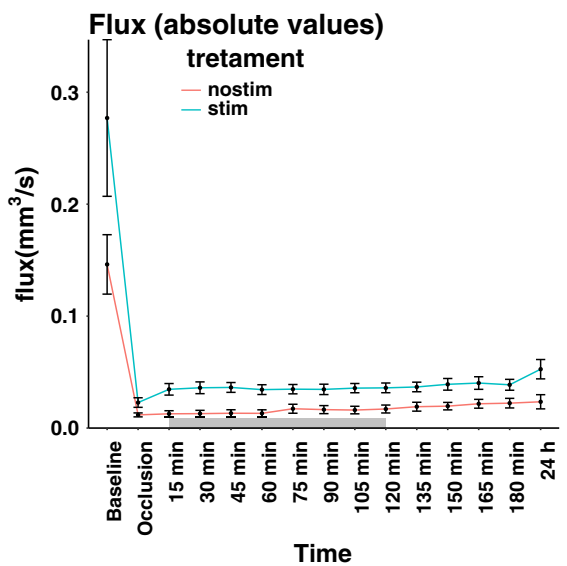

(d)

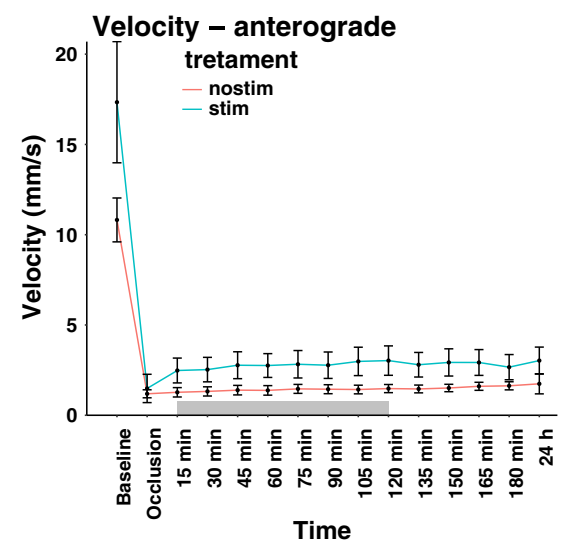

(b)

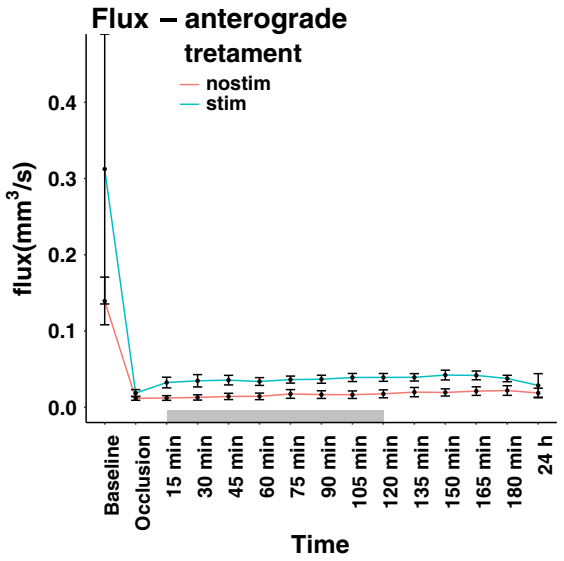

(e)

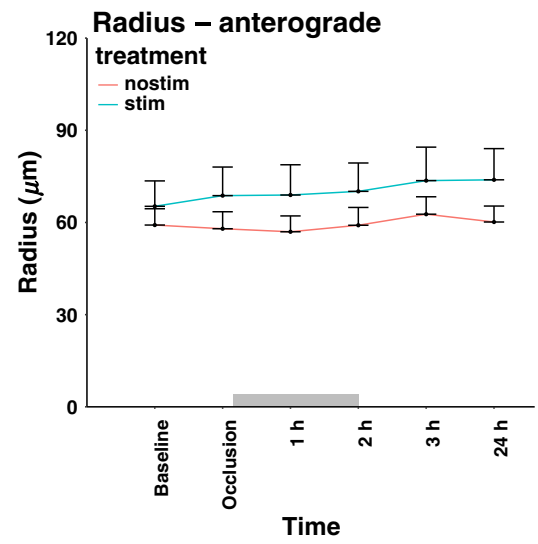

(g)

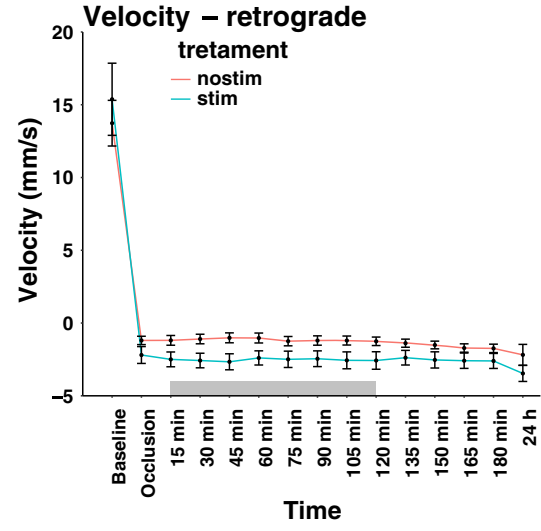

(c)

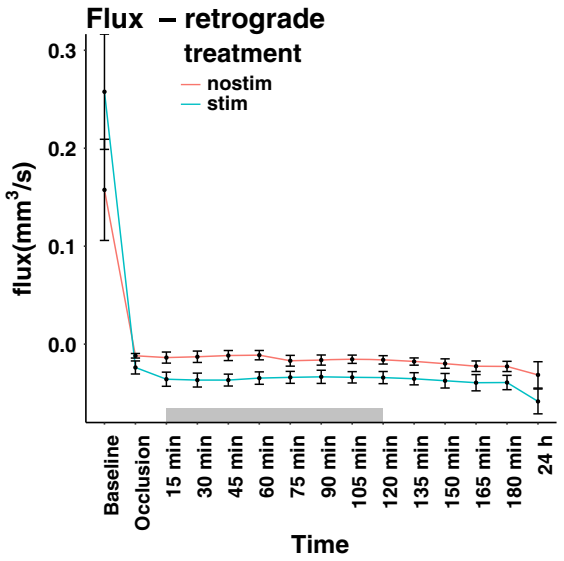

(f)

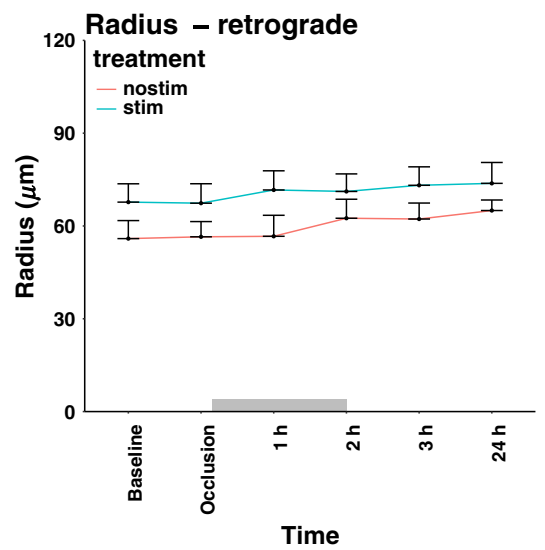

(h)

Fig. 6 Change in flow patterns in pMCAo groups ( $+0 \mathrm{~h}$ and no-stimulation) prior and following pMCAo and up to $24 \mathrm{~h}$. Changes are shown for stimulated (green) versus nonstimulated (red) branches for velocity: (a) absolute values, (b) anterograde branches, and (c) retrograde branches; flux: (d) absolute values, (e) anterograde branches, and (f) retrograde branches. For the radii (sampled in a lower rate), absolute values were not relevant, and therefore, only $(\mathrm{g})$ anterograde and $(\mathrm{h})$ retrograde branches are shown. Stimulus duration is denoted by a gray rectangle above the $x$-axis. No stim group is denoted in red; stim group is denoted in green. Note that "retrograde" and "anterograde" labels have no meaning before pMCAo. See text for details.

branches $\quad-0.007 \pm 0.003 \mathrm{~mm}^{3} / \mathrm{s} \quad(d f=10, \quad p=0.06)$. However, within the first $45 \mathrm{~min}$ following stimulation, the flux was stronger by $-0.011 \pm 0.004 \mathrm{~mm}^{3} / \mathrm{s}(d f=10, p=0.03)$, but this difference disappeared within the 60 to $180 \mathrm{~min}$ duration flowing occlusion where the difference became much smaller $-0.006 \pm 0.003 \mathrm{~mm}^{3} / \mathrm{s}(d f=10, p=0.12)$, Fig. 7(f). Finally, no differences were found between the absolute values of anterograde versus retrograde flux for the $+0 \mathrm{~h}$ $\left(-0.005 \pm 0.004 \mathrm{~mm}^{3} / \mathrm{s} ; d f=10, p=0.3\right)$ as well as for the no-stimulation flux $\left(-0.0011 \pm 0.003 \mathrm{~mm}^{3} / \mathrm{s} ; d f=11\right.$, 


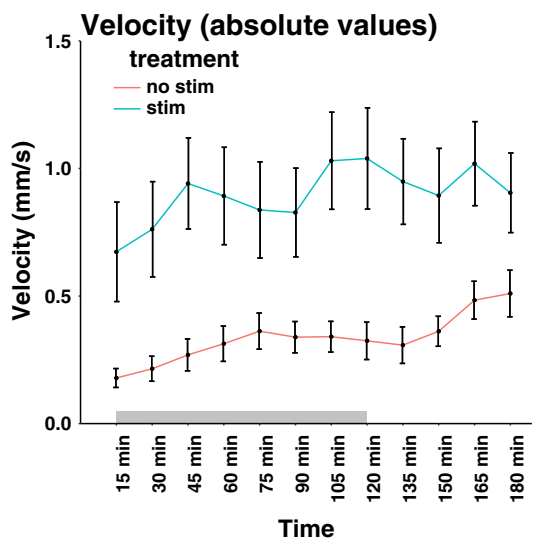

(a)

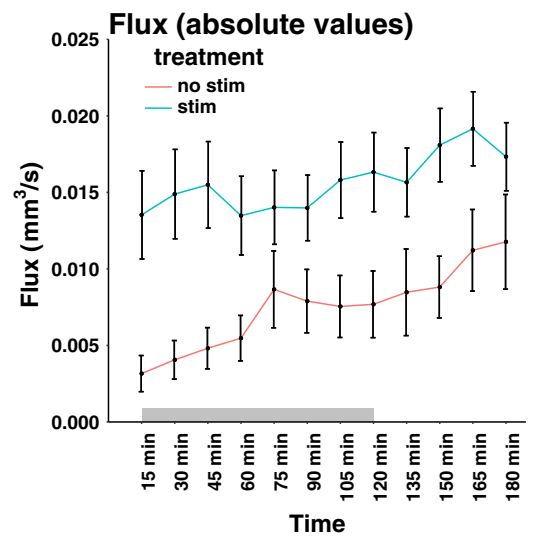

(d)

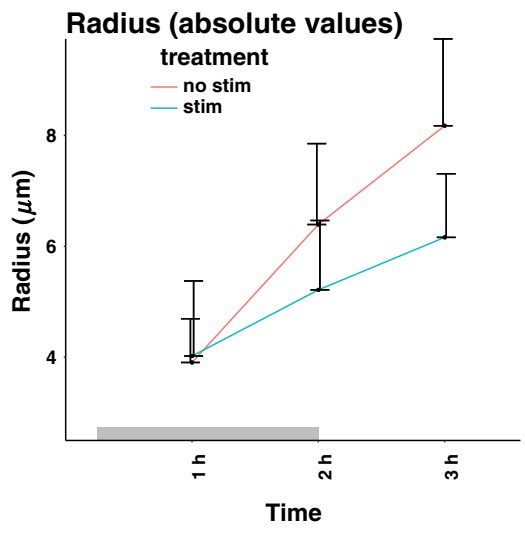

(g)

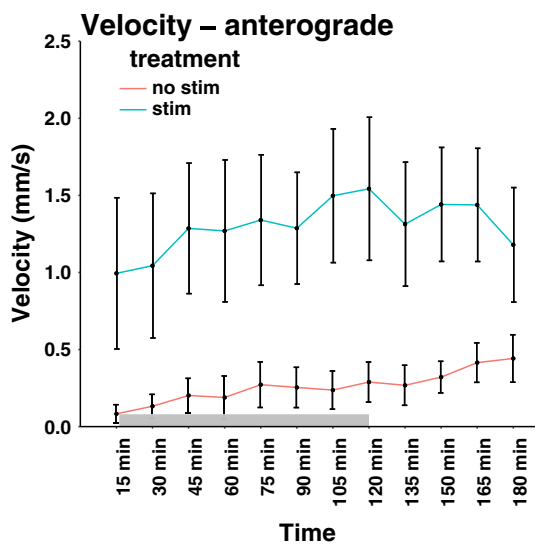

(b)

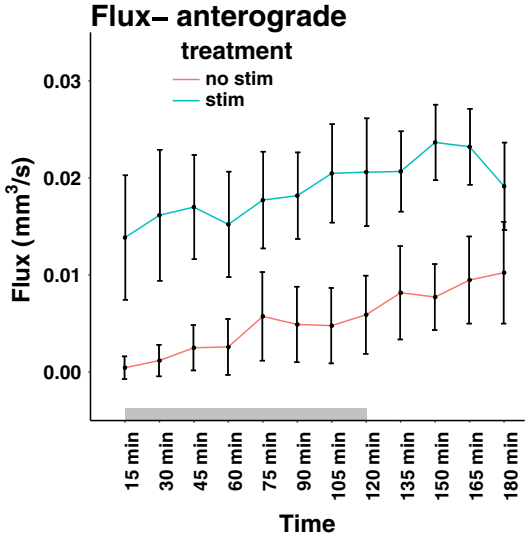

(e)

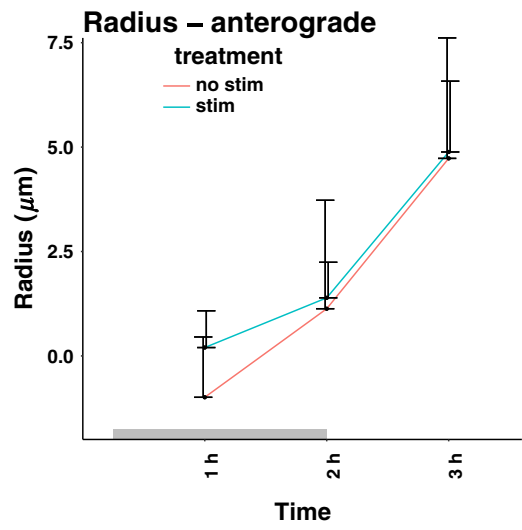

(h)

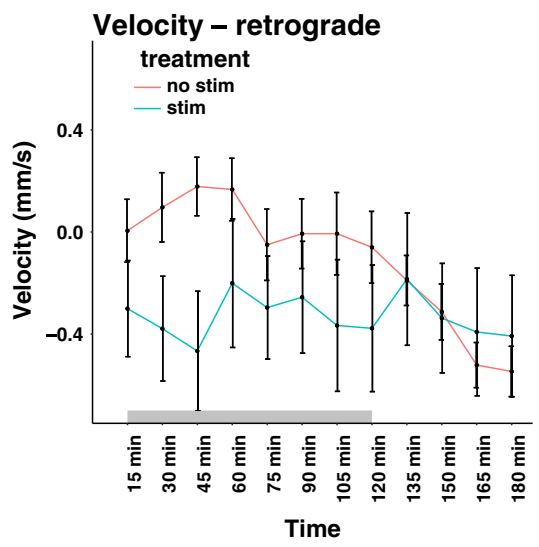

(c)

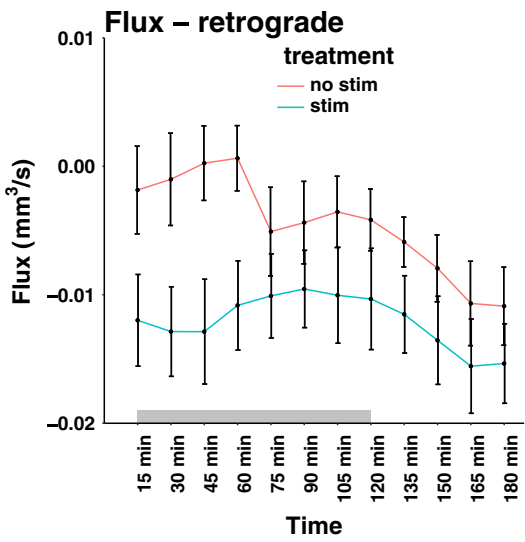

(f)

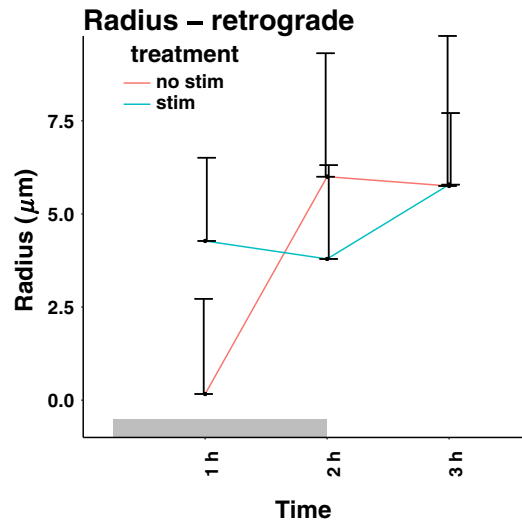

(i)

Fig. 7 Detailed analysis of difference in flow parameters and radii analyzed as differences from the occlusion point to $180 \mathrm{~min}$ in pMCAo groups ( $+0 \mathrm{~h}$ and no-stimulation) where colors denote stimulated (green) versus nonstimulated (red) branches. (a) Velocity (absolute values), (b) anterograde, and (c) retrograde. (d) Flux (absolute values), (e) anterograde, and (f) retrograde. (g) Radius (absolute values), (h) anterograde branches, and (i) retrograde branches. Stimulus duration is denoted by a gray rectangle above the $x$-axis. See text for details.

$p=0.73)$. Collectively, these findings show a clear general enhancing effect of the stimulation on flux. Like the velocity case, further analysis suggests that this effect can be mostly attributed to anterograde branches. When stimulation was present, anterograde flux was stronger in the stimulated branches versus the nonstimulated branches. For the retrograde branches, the analysis suggests a more complex situation where the presence of the stimulation had no clear mean effect on the flux, potentially due to an increase in variability, but was temporarily stronger within the first 45 min following occlusion.

Radii of the branches. For the pooled anterograde and retrograde branches' values, there was no difference in radii: $-1.03 \pm 1.48 \mu \mathrm{m}(d f=30, p=0.49)$ Fig. $7(\mathrm{~g})$. For anterograde blood flow $1.83 \pm 2.3 \mu \mathrm{m}(d f=11, p=0.4)$ [Fig. 7(h)] as well as for retrograde blood flow, there was no difference in 
means $\quad 5.73 \pm 5.70 \mu \mathrm{m} \quad(d f=10, \quad p=0.33) \quad$ [Fig. $\quad 7(\mathrm{i})]$. However, when the variable time was added to the regression formula (see Sec. 2.3.1), it was found that for anterograde blood flow the mean radius grew over time by $0.71 \pm 0.24 \mu \mathrm{m}$ $(d f=9, p=0.01)$, regardless of the presence of stimulation, Fig. 7(h). Finally, no differences were found between the absolute values for anterograde versus retrograde branches radii for the $+0 \mathrm{~h}(3.4 \pm 1.65 \mu \mathrm{m} ; t=-2.06, d f=10, p=0.06)$ or for the no-stimulation branches radii $(1.21 \pm 2.02 \mu \mathrm{m} ; t=-0.6$, $d f=11, p=0.56)$. Collectively, these results suggest that the radii of the vessels were not influenced by the presence of the stimulation in the experimental groups except for a significant trend to expand over time (from occlusion to $180 \mathrm{~min}$ ) in the anterograde group.

\subsubsection{Twenty-four hours following pMCAo}

When flow parameters were separately analyzed for the $24 \mathrm{~h}$ point, velocities increased from occlusion points to $24 \mathrm{~h}$ time points from 1.6 to $2.6 \mathrm{~mm} / \mathrm{s}$ ( $163.9 \%$ of occlusion), flux increased from 0.017 to $0.038 \mathrm{~mm}^{3} / \mathrm{s}(220.5 \%$ of occlusion), and radii increased from 62.8 to $68 \mu \mathrm{m}$ (108.3\% of occlusion). The analysis was further divided to separate experimental groups shown in Fig. 6. After assessing normality by ShapiroWilks normality tests, values of flow velocity were analyzed as compared to the occlusion point values by employing one-sided Welch $t$-tests to check if flow parameters were significantly higher at the 24-h time point compared to the occlusion time point. We found for anterograde no-stimulation a difference of $0.55 \pm 0.09 \mathrm{~mm} / \mathrm{s} \quad(p=0.035)$; for anterograde $+0 \mathrm{~h}$ $1.24 \pm 0.34 \mathrm{~mm} / \mathrm{s}(p=0.099)$, for retrograde $+0 \mathrm{~h}-1.27 \pm$ $0.16 \mathrm{~mm} / \mathrm{s}(p=0.017)$; since normality was not affirmed for retrograde no-stimulation, a Wilcoxon signed-rank test was conducted to find a difference of $-0.99 \pm 0.37 \mathrm{~mm} / \mathrm{s}(p=0.34)$.

The same analysis led to the following results for the flux values: since the assumption of normality was rejected in all cases by a series of Shapiro-Wilks tests, we employed one-sided Wilcoxon signed-rank tests to find differences for anterograde no-stimulation $0.007 \pm 0.0017 \mathrm{~mm}^{3} / \mathrm{s}(p=0.097)$; for anterograde $+0 \mathrm{~h} 0.101 \pm 0.0079 \mathrm{~mm}^{3} / \mathrm{s}(p=0.219)$; for retrograde $+0 \mathrm{~h}-0.035 \pm 0.0033 \mathrm{~mm}^{3} / \mathrm{s}(p=0.003)$; and for retrograde no-stimulation $-0.020 \pm 0.0053 \mathrm{~mm}^{3} / \mathrm{s}(p=0.078)$.

For the radii, the following results were found. Since the assumption of normality was affirmed in all cases by a series of Shapiro-Wilks tests, we employed two-sided Welch $t$-tests to check if the radii changed. For anterograde, no-stimulation $-2.2 \pm 7.6 \mu \mathrm{m}(d f=9, \quad p=0.55) ;$ for anterograde $+0 \mathrm{~h}$ $-5.2 \pm 13.8 \mu \mathrm{m}(d f=5, p=0.01) ;$ for retrograde $+0 \mathrm{~h}$ $-6.2 \pm 9.3 \mu \mathrm{m}(d f=10, p=0.04)$; and for retrograde no stimulation $-8.5 \pm 5.9 \mu \mathrm{m}(d f=5, p=0.07)$.

Collectively, these findings show that previously stimulated retrograde branches had an increased velocity and flux following $24 \mathrm{~h}$, but not for the other groups (except for the nonstimulated anterograde velocity). The increase in the radius of the $+0 \mathrm{~h}$ retrograde group could have directly contributed to these findings.

\subsubsection{DOCT imaging of slower flow}

In our measurements, flow velocities were quantified in major MCA branches of each subject using the Doppler phase method. In order to detect the relatively slower flow, the sampling A-line interval should be increased. However, increase of the sampling

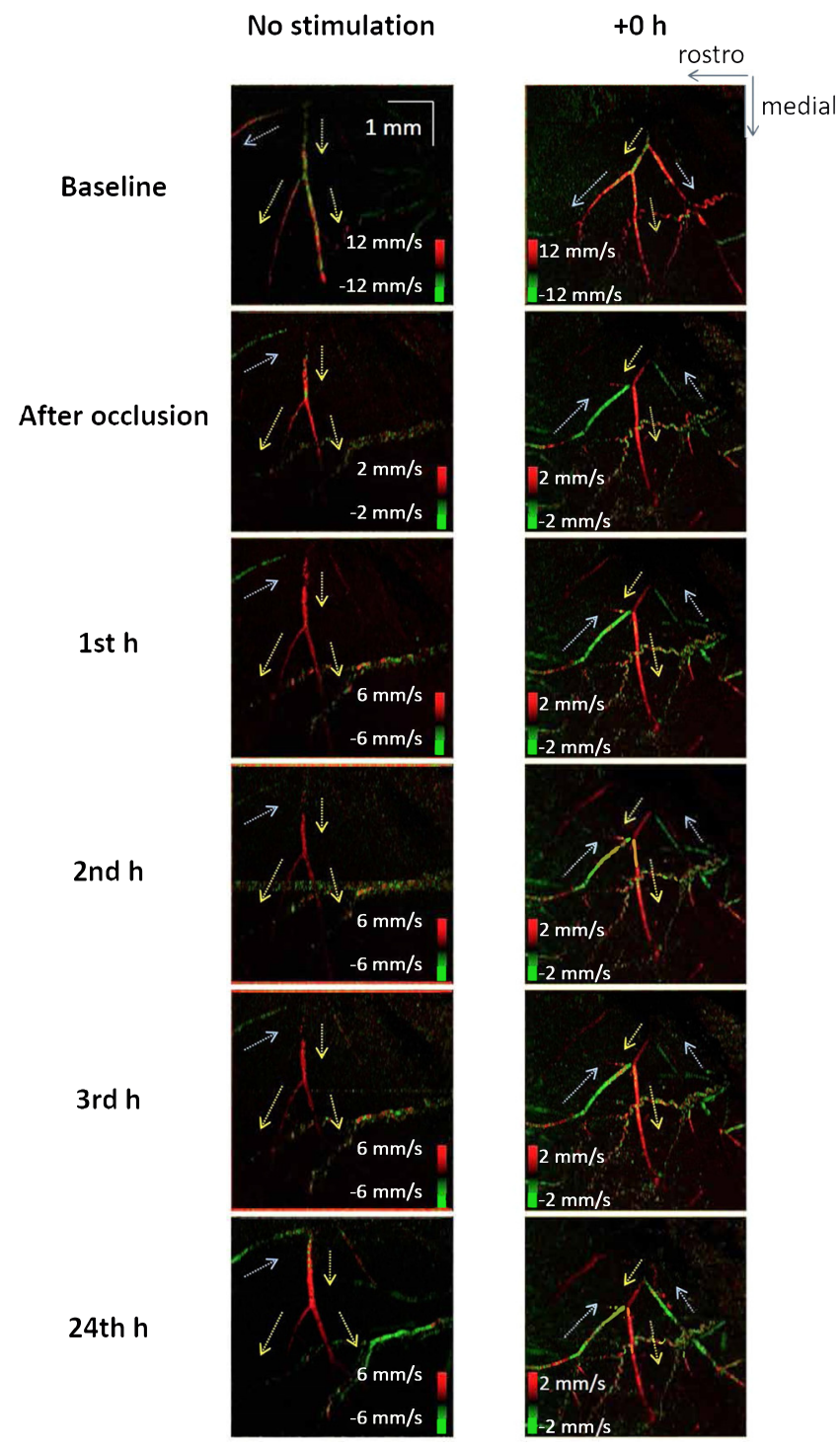

Fig. 8 Example of maximum intensity projection using a phaseresolved Doppler method. Blue arrows denote retrograde flow and yellow arrows denote anterograde flow. Note reversal of flow direction following occlusion in each example. The green points in the red branch and the red points in the green branch represent phase wrapping due to larger velocities.

interval results in phase wrapping and more noise due to phase instability. Therefore, for mapping of the microvascular networks, which include vessels with relatively slower flow, an intensity-based IBDV method was used with a high sampling interval of $2.0 \mathrm{~ms}$. Though the IBDV method cannot quantify the accurate velocities and cannot determine their flow directions, more flow information about the microvascular networks can be revealed by combining it with the Doppler phase images. The vascular networks in a $+0 \mathrm{~h}$ subject (the same subject as shown in Fig. 8 right column) at baseline, 3, and $24 \mathrm{~h}$ are shown in Fig. 9. Due to the higher sampling A-line interval, the vessels with slower flow could also be imaged. Notably, after occlusion, the blood flow was strongly reduced in the far dorsal parts of major MCA branches as indicated by the green arrows, yet flow remained sustained in the more proximal parts of the same MCA branches as indicated by blue arrows. Figure 9 shows changes in 
Baseline

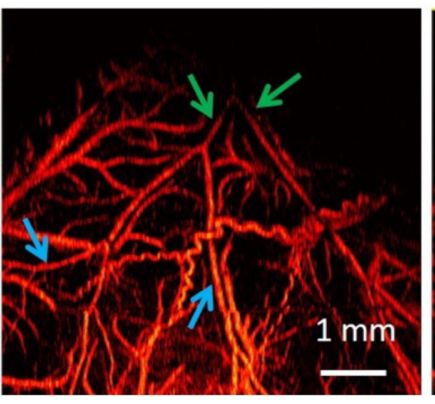

$3 \mathrm{~h}$ after occlusion

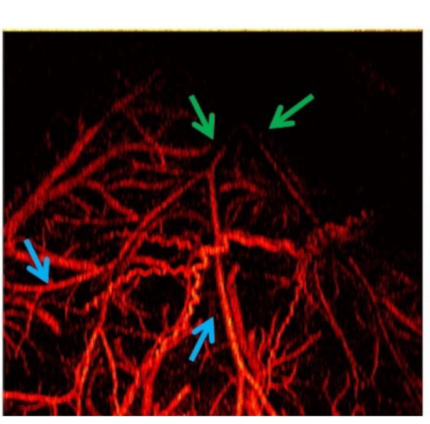

$24 \mathrm{~h}$ after occlusion

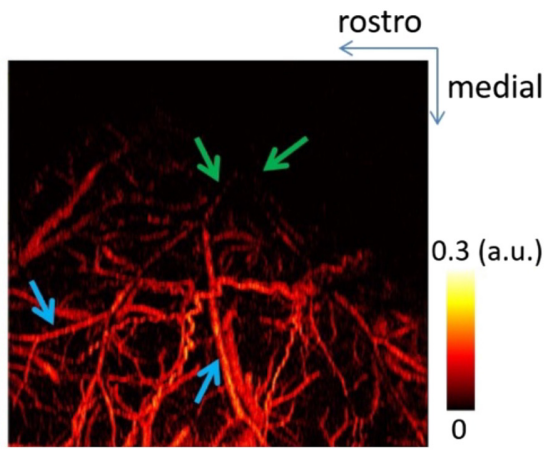

Fig. 9 En face projections of IBDV images of vascular networks in the MCA region of a $+0 \mathrm{~h}$ subject. A sampling A-line interval of $2.00 \mathrm{~ms}$ was used to highlight blood vessels with slower flow speed. After occlusion, there was a major reduction in blood flow speed in the far dorsal parts of major MCA branches as indicated by the green arrows, yet flow speed remained sustained in the more proximal parts of the same MCA branches as indicated by blue arrows.

the largest MCA vessels and no changes in the small vessels that are supplied by the large branches probably because retrograde blood flow was present in the MCA branches and there were additional sources providing blood flow into the distal MCA branches, as shown in Fig. 8 (the $+0 \mathrm{~h}$ case).

\subsubsection{Outliers}

Notably, velocities in most MCA branches decreased to $10 \%$ to $12 \%$ of baseline values following pMCAo. We found, however, two exceptional cases in which blood flow became immediately retrograde and remained at surprisingly high velocity levels. In one stimulated branch, flow velocity remained at $100 \%$ baseline value and at $78 \%$ baseline value in another no-stimulation branch. The first value was found to be an outlier and that branch was excluded from analyses. Another branch showing reversal of flow parameters in the middle of the stimulation period was also excluded from the analysis.

\subsubsection{Histology}

Edema-corrected TTC analysis was blindly assessed. The analysis showed that the sham surgery group had minimal mean damage of $1.29 \pm 0.26 \mathrm{~mm}^{3}$; the stimulated $(+0 \mathrm{~h})$ group mean volume damage was $8.79 \pm 1.19 \mathrm{~mm}^{3}$, and for the nonstimulated group, the mean volume damage was $29.31 \pm 3.95 \mathrm{~mm}^{3}$. The nonstimulated group damage volume was larger than the +0 h group (one-way, $t=1.9, d f=6, p=0.04$ ).

\section{Discussion}

The application of DOCT revealed several unexpected findings and insights regarding the spatiotemporal characteristics of the pial collateral-dorsal MCA system in our anesthetized rat pMCAo model. The ability of DOCT to inform about the direction of flow revealed the existence of branches with anterograde flow following pMCAo in almost every imaged subject. This finding suggests the hypothesis that not every major dorsal MCA branch has a functional collateral, otherwise we would expect to image only retrograde flow in all the imaged branches. Indeed, previous flow imaging using LSI-a technique that is agnostic to blood flow direction-led to the impression that collateral flow following pMCAo, within the same MCA branches as analyzed here, is always retrograde because in our pMCAo model the MCA is severed, and therefore, there is no clear known source for anterograde blood flow. Indeed, pMCAo in addition to permanent occlusion of the dorsal MCA branches near the collaterals abolished the ability for sensory-based protection. ${ }^{11}$ Further, in this study, there was no clear way to predict which MCA branch has a functional collateral because similar MCA branches in different subjects could have either anterograde or retrograde flow. Such heterogeneity was revealed despite the fact that all subjects in this study were purchased from the same building in our traditional breeder's facility. Surprisingly, in some subjects, only a single MCA branch showed retrograde flow whereas all other imaged branches showed anterograde flow. Further, few branches showed a change in blood flow direction during the stimulation period or at the 24-h time point, suggesting the collateral system can exhibit dynamic properties because a blood flow in a specific direction is not always guaranteed over time. Finally, the unexpected directions of blood flow patterns in major MCA branches and their dynamics could also potentially explain similar findings regarding different directions of flow in smaller surface arterioles following temporary MCAo, ${ }^{39,40}$ arterioles that are fed by the large MCA branches studied here, and therefore, their different directions of flow could be explained by the different directions of flow in the MCA major branches that feed them. However, as these studies and our study differ in the type of anesthesia and the way MCAo has been induced, such comparison among these studies should be cautiously interpreted.

Not only did we find vessels with anterograde velocity and flux, the presence of sensory stimulation was more effective in the anterograde flow vessels compared to the retrograde flow vessels. The anterograde flow showed clear increase in flow parameters (velocity, flux) in the presence of stimulation and up to $180 \mathrm{~min}$ ( $1 \mathrm{~h}$ after stimulus termination) despite a clear increase in variability. However, for the retrograde flow branches, the $2 \mathrm{~h}$ stimulus had only a transient effect on the flux for the first $45 \mathrm{~min}$ following occlusion. Further research is needed to reveal why the retrograde branches show this rapid adaptation to the stimulation presence whereas the anterograde branches show faster velocity and stronger flux in the presence of stimulation and even $1 \mathrm{~h}$ beyond stimulus termination. These findings, however, led to a conundrum. We assumed that the retrograde flow is the only potential source of the anterograde flow [Fig. 10(a)], but that would imply that the retrograde and anterograde flows should 

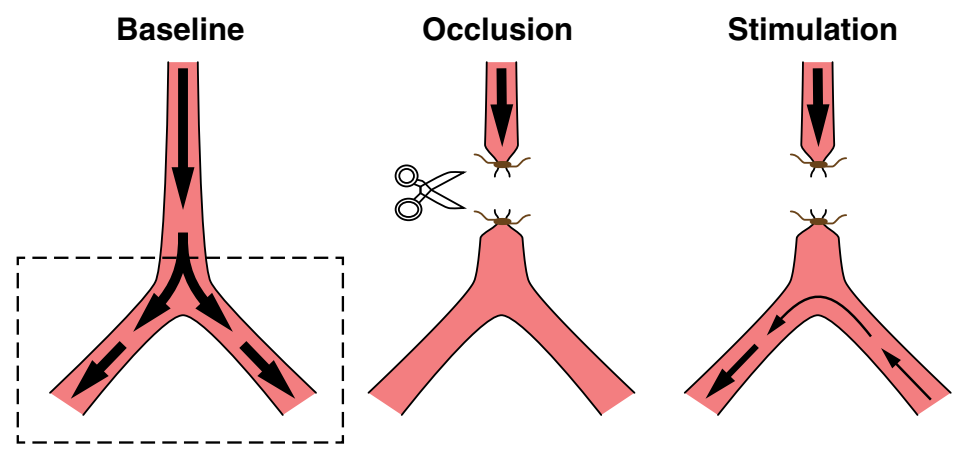

(a)
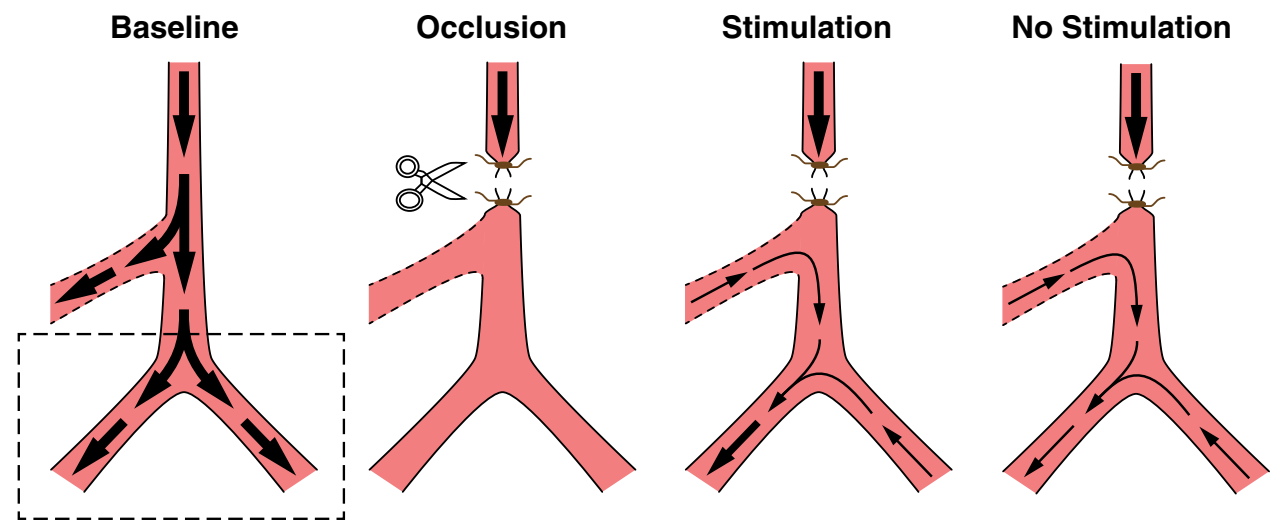

(b)

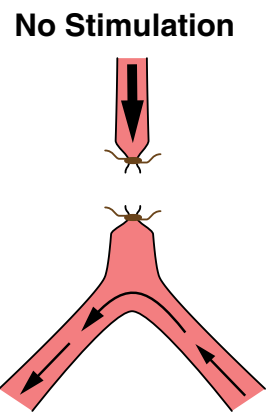

Fig. 10 Cartoon of the main the dorsal MCA branches and their blood flow patterns before and after pMCAo. (a) Intuitive explanation of our findings. Two main dorsal MCA branches are imaged by DOCT (imaged area is denoted by a rectangle on the left) with strong natural anterograde flow (wide, bold arrows). Following occlusion, retrograde flow in a branch that is connected to functional collateral supplies blood to the anterograde branch. In the presence of sensory stimulation, the anterograde flow becomes stronger than the retrograde flow as denoted by the width of the arrows. However, it is not clear how the retrograde and anterograde flows can show different flow patterns if the former is the only provider for the latter. Therefore, in (b) a different hypothesis is shown. In this case, there is a third MCA branch (denoted by dashed lines) outside the imaged area (denoted by the rectangle). Following pMCAo, retrograde flow in the unimaged branch supplies additional blood flow to the anterograde branch, explaining the discrepancy between retrograde and anterograde branches shown in (a). See text for details on another, complementary hypothesis about the increase in radius of anterograde vessels over time.

have similar flow parameters, which clearly was not the case. Our current hypothesis is that our imaging window may not have been large enough to allow imaging of potential additional MCA branch(es) with retrograde flow following pMCAo, and that these unimaged branch(es) directly supplied additional flow to the anterograde flow branches [Fig. 10(b)]; see also Fig. 1(b) for an example of a case of a major MCA branch that is clearly outside the DOCT imaging window. Alternatively, another potential contribution to the anterograde dominance is the constant increase in the radius of anterograde branches over time [Fig. 7(h)]. The unimaged branch hypothesis and the increase in radius hypothesis are not mutually exclusive and could work together. We therefore suggest that future DOCT imaging experiments should employ a much larger imaging window that should include all dorsal MCA branches. Another useful approach would be to employ DOCT imaging to directly capture all MCA collaterals, as was previously done using LSI. ${ }^{41}$

Despite the stronger and more persistent influence of sensory stimulation on anterograde branches during the period between the occlusion to 180 -min time points, at $24 \mathrm{~h}$, the results were mostly opposite. At the 24-h time point, when compared with the occlusion point, both velocity and flux of the previously stimulated retrograde branches were faster and stronger, respectively, potentially at least partially explained by the increase in radius of this group. Thus, despite the only transient effect of protective stimulation on retrograde branches blood flow parameters in the longer run (i.e., at $24 \mathrm{~h}$ ), the retrograde flow parameters prevailed over the anterograde flow parameters. These findings highlight again the dynamic temporal nature of the collaterals-dorsal MCA branches system.

Our findings indicate that cortex can be protected despite extremely low levels of velocity and flux following pMCAo. These findings provide evidence for the unusual ability of the cortical neurovascular and autoregulation systems to adapt and survive even at extremely low flow levels. Even at the 24-h time point, velocity and flux increase was only modest compared to the occlusion reference point and still small when compared to the preocclusion baseline. However, it is important to keep in mind that other variables such as oxygen availability could also be critical for tissue viability. Thus, for example, sensory-based treatment could also result in improved oxygen partial pressure $\left(\mathrm{SpO}_{2}\right)$ and oxygen saturation $\left(\mathrm{StO}_{2}\right)$ compared 
to no-stimulation controls, despite having very low blood flow velocity and flux. We have previously shown that arterial oxygen saturation is maintained in treated subjects ${ }^{12}$ but falls in nontreated subjects; ${ }^{42}$ and therefore, further studies of these parameters within the same subject are clearly warranted.

Our findings are qualitatively congruent with our previous LSI results but differ quantitatively. Qualitatively, employing both blood flow imaging techniques, a major reduction was demonstrated following pMCAo, stimulation enhanced faster flow velocity versus nonstimulated vessels, and there was rebound in velocity at 24-h time point. Quantitatively, however, the major reduction in velocity following pMCAo for DOCT was less than half of the LSI results (DOCT: to $11.4 \%$ of baseline velocity; to $8.17 \%$ of baseline flux. LSI: to $26 \%$ of baseline velocity even after correction for dead brain values). ${ }^{12}$ The quantitative discrepancy, starting to build-up following occlusion, is especially evident at the 24-h time point where LSI has demonstrated a return to preocclusion baseline levels in protected animals, ${ }^{11}$ a finding that is not supported by the current results that showed a less impressive increase toward preocclusion baseline in velocity and flux at the 24-h time point [Fig. 6(a)]. The quantitative discrepancy between the imaging methods could be related to the fact that the LSI's laser beam samples not only from a specific surface vessel, but the measurement can also be influenced by neighboring parenchyma tissue, especially from tissue located beneath the imaged vessel, whereas DOCT measures flow parameters only from the vessel itself in a matter akin to confocal microscopy. However, DOCT measurement at $24 \mathrm{~h}$ should also be cautiously interpreted as this technique could be quite sensitive to minor changes in the adjustment of the imaged cortex in relation to the angle of the laser beam when the animal is repoisoned under the DOCT imaging setup. Alternatively, although unlikely, we cannot rule out the hypothesis that our previous LSI flow measurements, obtained from single MCA dorsal branches, were acquired from branches that already showed $80 \%$ or $100 \%$ retrograde flow after occlusion and beyond, as two branches showed in our current study.

The dynamic nature of the collateral-MCA system combined with the unpredictability regarding the identity of branches that show anterograde flow versus retrograde flow presents a new variable that potentially could influence our understanding of collateral-based MCA flow and its impact on the final outcome of a typical pMCAo experiment. With the growing recognition of the importance of collateral flow in preclinical and clinical studies, our findings indicate a growing need for further, direct imaging studies of the pial collateral system. Unfortunately, our findings may not be generalizable to other rodents as in our hands, sensory-based protection seems to be ineffective in mice, potentially due to minimal collateral flow as imaged 24-h postpMCAo that did not change in the presence of stimulation, ${ }^{43}$ and therefore, rats probably remain a more optimal target for further studies of the collateral system. Of special interest should be the study of the collateral system in cases of comorbidity. We have demonstrated that sensory-based protection is as effective in old rats as it is in young adults, ${ }^{14}$ but is ineffective in spontaneously hypertensive rats. ${ }^{44}$ In the latter case, we found that despite normal blood flow values before pMCAo, the collateral flow was minimal following 24-h post-pMCAo and did not change in the presence of stimulation, which resulted in a very large volume of stroke damage that typically seemed to include the entire MCA territory.
In conclusion, this study suggests that the dorsal MCA main branches have an unpredictable probability of being connected to a functional collateral that, in turn, dictates flow patterns (anterograde versus retrograde) within the dorsal branches. Further, MCA dorsal branches show unique spatiotemporal dynamics in their flow patterns and in their response to the presence of stimulation. Sensory stimulation enhanced flow parameters, transiently in retrograde branches, and more strongly and with longer durability in anterograde branches, but the previously stimulated retrograde flow branches showed enhanced velocity and flux at 24-h following occlusion.

\section{Disclosures}

Dr. Zhongping Chen has a financial interest in OCT Medical Imaging, Inc., which, however, did not support this work. All other authors declare no conflict of interest, financial or otherwise.

\section{Acknowledgments}

This work was supported by the U.S National Institutes of Health under Grant Nos. R01HL-125084, R01HL-127271, R01EY-026091, R01EY-021529, and P41EB-015890 to Dr. Zhongping Chen, and Grant No. NS-066001 and the Leducq Foundation Grant No. 15CVD02 to Dr. Ron D. Frostig. We also thank Gabriel Hui for creating Fig. 10.

\section{References}

1. D. Mozaffarian et al., "Heart disease and stroke statistics-2016 update: a report from the American Heart Association," Circulation 133(4), e38-e360 (2016).

2. R. Bhatia et al., "Low rates of acute recanalization with intravenous recombinant tissue plasminogen activator in ischemic stroke: real-world experience and a call for action," Stroke 41(10), 2254-2258 (2010).

3. M. Goyal et al., "Randomized assessment of rapid endovascular treatment of ischemic stroke," N. Engl. J. Med. 372(11), 1019-1030 (2015).

4. A. J. Yoo and T. Andersson, "Thrombectomy in acute ischemic stroke: challenges to procedural success," J. Stroke 19(2), 121-130 (2017).

5. M. G. Burnett et al., "Electrical forepaw stimulation during reversible forebrain ischemia decreases infarct volume," Stroke 37(5), 1327-1331 (2006).

6. A. Bandla et al., "Peripheral sensory stimulation is neuroprotective in a rat photothrombotic ischemic stroke model," in 2016 38th Ann. Int. Conf. of the IEEE Eng. in Med. and Biol. Soc., pp. 6086-6089 (2016).

7. L. D. Liao et al., "Improving neurovascular outcomes with bilateral forepaw stimulation in a rat photothrombotic ischemic stroke model," Neurophotonics 1(1), 011007 (2014).

8. L. D. Liao et al., "Rescue of cortical neurovascular functions during the hyperacute phase of ischemia by peripheral sensory stimulation," Neurobiol. Dis. 75, 53-63 (2015).

9. T. Guo et al., "Pulsed transcranial ultrasound stimulation immediately after the ischemic brain injury is neuroprotective," IEEE Trans. Biomed. Eng. 62(10), 2352-2357 (2015).

10. H. C. Pan et al., "Neurovascular function recovery after focal ischemic stroke by enhancing cerebral collateral circulation via peripheral stimulation-mediated interarterial anastomosis," Neurophotonics 4(3), 035003 (2017).

11. C. C. Lay et al., "Mild sensory stimulation completely protects the adult rodent cortex from ischemic stroke," PLoS One 5(6), e11270 (2010).

12. C. C. Lay et al., "Mild sensory stimulation reestablishes cortical function during the acute phase of ischemia," J. Neurosci. 31(32), 1149511504 (2011).

13. M. F. Davis et al., "Amount but not pattern of protective sensory stimulation alters recovery after permanent middle cerebral artery occlusion," Stroke 42(3), 792-798 (2011).

14. C. C. Lay et al., "Mild sensory stimulation protects the aged rodent from cortical ischemic stroke after permanent middle cerebral artery occlusion," J. Am. Heart Assoc. 1(4), e001255 (2012). 
15. M. F. Davis, C. Lay, and R. D. Frostig, "Permanent cerebral vessel occlusion via double ligature and transection," J. Vis. Exp. 77, 1-8 (2013).

16. C. C. Lay et al., "Early stimulation treatment provides complete sensory-induced protection from ischemic stroke under isoflurane anesthesia," Eur. J. Neurosci. 38, 2445-2452 (2013).

17. A. M. Hancock et al., "Sensory stimulation-based complete protection from ischemic stroke remains stable at 4 months post-occlusion of MCA," J. Neurol. Disord. 1(4), 135 (2013).

18. C. C. Lay and R. D. Frostig, "Complete protection from impending stroke following permanent middle cerebral artery occlusion in awake, behaving rats," Eur. J. Neurosci. 40(9), 3413-3421 (2014).

19. R. D. Frostig, C. C. Lay, and M. F. Davis, "A rat's whiskers point the way toward a novel stimulus-dependent, protective stroke therapy," Neuroscientist 19, 313-328 (2013).

20. J. C. Baron, "Protecting the ischaemic penumbra as an adjunct to thrombectomy for acute stroke," Nat. Rev. Neurol. 14(6), 325-337 (2018).

21. D. von Bornstadt et al., "Sensory stimulation in acute stroke therapy," J. Cereb. Blood Flow Metab. 38(10), 1682-1689 (2018).

22. A. Shuaib et al., "Collateral blood vessels in acute ischaemic stroke: a potential therapeutic target," Lancet Neurol. 10(10), 909-921 (2011).

23. S. A. Sheth and D. S. Liebeskind, "Imaging evaluation of collaterals in the brain: physiology and clinical translation," Curr. Radiol. Rep. 2(1), 29 (2014).

24. I. R. Winship, "Cerebral collaterals and collateral therapeutics for acute ischemic stroke," Microcirculation 22(3), 228-236 (2015).

25. X. Leng et al., "Impact of collateral status on successful revascularization in endovascular treatment: a systematic review and meta-analysis," Cerebrovasc. Dis. 41(1-2), 27-34 (2016).

26. M. D. Ginsberg, "Expanding the concept of neuroprotection for acute ischemic stroke: the pivotal roles of reperfusion and the collateral circulation," Prog. Neurobiol. 145-146, 46-77 (2016).

27. G. W. Albers et al., "Thrombectomy for stroke at 6 to 16 hours with selection by perfusion imaging," N. Engl. J. Med. 378, 708-718 (2018).

28. A. Vagal et al., "Collateral clock is more important than time clock for tissue fate: a natural history study of acute ischemic strokes," Stroke $\mathbf{4 9}$, 2102-2107 (2018).

29. Z. Chen and J. Zhang, "Doppler optical coherence tomography," in Optical Coherence Tomography: Technology and Application, W. Drexler and J. G. Fujimoto, Eds., pp. 52-67, Springer International Publishing, Cham (2015).

30. Z. Chen and J. Zhu, "Doppler optical coherence tomography and its application in measurement of cerebral blood flow," in Neurophotonics and Brain Mapping, Y. Chen and B. Kateb, Eds., pp. 159-176, Taylor \& Francis, Boca Raton, Florida (2017).
31. Q. Li et al., "Fully distributed absolute blood flow velocity measurement for middle cerebral arteries using Doppler optical coherence tomography," Biomed. Opt. Express 7(2), 601-615 (2016).

32. J. B. Bederson et al., "Evaluation of 2, 3, 5-triphenyltetrazolium chloride as a stain for detection and quantification of experimental cerebral infarction in rats," Stroke 17(6), 1304-1308 (1986).

33. Y. Zhao et al., "Phase-resolved optical coherence tomography and optical Doppler tomography for imaging blood flow in human skin with fast scanning speed and high velocity sensitivity," Opt. Lett. 25(2), 114-116 (2000).

34. Y. Zhao et al., "Doppler standard deviation imaging for clinical monitoring of in vivo human skin blood flow," Opt. Lett. 25(18), 1358-1360 (2000).

35. G. Liu et al., "Intensity-based modified Doppler variance algorithm: application to phase instable and phase stable optical coherence tomography systems," Opt. Express 19(12), 11429-11440 (2011).

36. M. Arellano, "Practitioners' corner: computing robust standard errors for within-groups estimators," Oxford Bull. Econ. Stat. 49(4), 431434 (1987).

37. K. Y. Liang and S. L. Zeger, "Longitudinal data analysis using generalized linear models," Biometrika 73(1), 13-22 (1986).

38. J. Pustejovsky, "Cluster-robust (sandwich) variance estimators with small-sample corrections," R package version 0.3.5, 2019, https:// CRAN.R-project.org/package=clubSandwich.

39. C. B. Schaffer et al., "Two-photon imaging of cortical surface microvessels reveals a robust redistribution in blood flow after vascular occlusion," PLoS Biol. 4(2), e22 (2006).

40. A. Y. Shih et al., "Active dilation of penetrating arterioles restores red blood cell flux to penumbral neocortex after focal stroke," J. Cereb. Blood Flow Metab. 29(4), 738-751 (2009).

41. G. A. Armitage et al., "Laser speckle contrast imaging of collateral blood flow during acute ischemic stroke," J. Cereb. Blood Flow Metab. 30(8), 1432-1436 (2010).

42. D. Abookasis et al., "Imaging cortical absorption, scattering, and hemodynamic response during ischemic stroke using spatially modulated near-infrared illumination," J. Biomed. Opt. 14(2), 024033 (2009).

43. A. M. Hancock and R. D. Frostig, "Testing the effects of sensory stimulation as a collateral-based therapeutic for ischemic stroke in C57BL/6J and CD1 mouse strains," PLoS One 12(9), e0183909 (2017).

44. A. M. Hancock and R. D. Frostig, "Hypertension prevents a sensory stimulation-based collateral therapeutic from protecting the cortex from impending ischemic stroke damage in a spontaneously hypersensitive rat model," PLoS One 13(10), e0206291 (2018).

Biographies of the authors are not available. 